\title{
Sulla trasformazione delle equazioni lineari, omogenee, a derivate parziali, con coefficienti costanti.
}

\author{
(Di Carlo Somiglana, a Pavia.)
}

\begin{abstract}
L。 in generale la trasformata di una equazione lineare, omogenea, a derivate parziali, con coefficienti costanti, quando alle variabili indipendenti si sostituiscono nuove variabili, legate alle prime da relazioni qualsivogliano, e dànno per la trasformata una nuova equazione lineare, in generale non più omogenea, nè a coefficienti costanti. Questi procedimenti però sono soggetti a due inconvenienti; in primo luogo conducono, specialmente per le equazioni di ordine elevato, a calcoli assai laboriosi, ed in secondo luogo lasciano completamente nell'ombra le relazioni, che pure devono esistere fra i coefficienti dell'equazione trasformata, poichè questa non è generale, ma gode evidentemente delle proprietà di essere, mediante la trasformazione inversa, ridotta omogenea, a coefficienti costanti. Tali relazioni poi sono di grande importanza, quando si tratta della integrazione delle equazioni; difatti, per citare un esempio notissimo, si sa come la forma concisa che si può dare alla trasformata della equazione di LAPLACE, in forza appunto di queste relazioni, permetta in molti casi di assegnarne degli integrali particolari con grandissima facilità.

La trasformazione della equazione di LAPLACE, 0 più in generale delle equazioni lineari, omogenee, a derivate parzıali, del $2 .^{\circ}$ ordine, con coefficienti costanti è stata oggetto di molte ricerche. Basti citare CAUCHY, LAMÉ e $\mathrm{J}_{\triangle \mathrm{COBI}}$ fra i primi che si occuparono della questione; e, dei moderni, BrIoschI (*)
\end{abstract}

(*) Teorica dei determinanti, § 10. Pavia, 1854.

Annali di Matematica, tomo XVIII. 
e specialmente Beltrami (*), che portò alla sua attuale generalità la teoria dei parametri differenziali, la quale comprende, come caso speciale, quella della trasformazione. È notissimo quale sia il risultato di queste ricerche, cioè che la trasformazione della equazione si può ridurre a quella di una forma differenziale quadratica, evitando cosi il calcolo diretto delle derivate di $2 .^{\circ}$ ordine delle variabili di un sistema rispetto a quelle dell'altro.

Ora rispetto alle equazioni di ordine superiore al secondo, per quanto so, non sono state stabilite ricerche analoghe a quelle ora ricordate, nè si ̀̀ cercato se $\mathrm{i}$ risultati ottenuti per quelle di $2 .^{\circ}$ ordine si potessero estendere ad equazioni di ordine superiore, quantunque queste, come la equazione di $\mathrm{LA}_{\mathrm{A}}$ PLACE, sebbene più raramente, si presentino in diversi problemi di fisica matematica. È questa appunto la questione, che mi sono proposto di trattare, e di cui, come si vedrà, mi pare di aver dato in via generale la soluzione.

Il problema della trasformazione si connette essenzialmente con un altro pure assai interessante: la determinazione di quelle espressioni invariabili rispetto ad una forma differenziale, che, estendendo la denominazione introdotta dapprima da LAMḱ, si possono chiamare parametri differenziali, anche quando la forma differenziale è di ordine superiore al secondo.

Nell' ultimo paragrafo di questo lavoro, generalizzando un teorema di Christorrer, sul quale si può dire fondato un metodo elegantissimo dovuto al prof. Ricor per costruire i parametri differenziali delle forme quadratiche $\left(^{* *}\right)$, ho ridotto la ricerca dei parametri delle forme di grado qualunque a quella degli invarianti algebrici simultanei di certi sistemi di forme differenziali, analogamente a quanto il prof. Ricci ha fatto per le forme quadratiche. Il problema algebrico essendo di gran lunga più semplice, e potendo in generale essere risoluto, ne viene che nello stesso modo anche la formazione dei parametri delle forme differenziali di grado qualsiasi potrà considerarsi, entro certi limiti, come un problema risoluto. Come applicazione del metodo generale ho trovato la espressione effettiva, per le forme del $4 .^{\circ}$ grado, di un gruppo di parametri che si presentano in certo modo come $\mathrm{i}$ corrispondenti di quelli noti delle forme quadratiche.

(*) Sulla teorica generale dei parametri differensiali. Accademia delle Scienze dell'Istituto di Bologna, 1869.

(**) RICcI, Sui parametri e gli invarianti delle forme quadratiche differenziali (Annali di Mat., S. 2. ${ }^{2}$, T. 14). - Sulla derivazione covariante ad una forma quadratica differenziale (Rend. della $R$. Acc. dei Lincei, 1887). 


\section{$\S 1$. Principio di Jacobi.}

Il metodo più semplice ed elegante che si conosca per la trasformazione dell'equazione di LAPLACE è quello che è stato dato da J J Ueber eine particuläre Lösung der partiellen Differentialgleichung $\frac{\partial^{2} V}{\partial x^{2}}+$ $+\frac{\partial^{2} V}{\partial y^{2}}+\frac{\partial^{2} V}{\partial z^{2}}=0$ (Crelle, Bd. 36, G. W., Bd. 2) e che è fondato sopra un principio di trasformazione che egli deduce dal calcolo delle variazioni. Questo principio non è esclusivamente applicabile alle equazioni di $2 .^{\circ}$ ordine, ma possiede una assai maggiore generalità. Noi, traducendo in formule l'enunciato

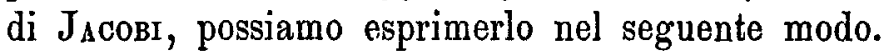

Si abbia una espressione $F^{\prime}$ formata in modo qualunque con una funzione $U$ di $n$ variabili $x_{1}, x_{2}, \ldots x_{n}$ e colle sue derivate parziali fino a quelle di un certo ordine; costruiamo l'espressione:

$$
G=\frac{\partial F}{\partial U}-\underset{i}{\mathbf{\Sigma}} \frac{\partial}{\partial x_{i}}\left(\frac{\partial F^{\prime}}{\partial\left(\frac{\partial U}{\partial x_{i}}\right)}\right)+\sum_{i s} \frac{\partial^{2}}{\partial x_{i} \partial x_{s}}\left(\frac{\partial^{2} F}{\partial\left(\frac{\partial^{2} L}{\partial x_{i} \partial x_{s}}\right)}\right)-\cdots
$$

e supponiamo che della $F$ si conosca la trasformata $\Phi$ in un certo sistema di variabili $y_{1}, y_{2}, \ldots y_{n}$, che sarà una funzione di $U$ e delle sue derivate parziali rispetto alle $y_{1}, y_{2}, \ldots y_{n}$. Se ora si pone:

$$
\Gamma=\frac{1}{\Delta} \frac{\partial \Phi}{\partial U}-\frac{1}{\Delta} \underset{i}{\mathbf{\Sigma}} \frac{\partial}{\partial y_{i}}\left(\Delta \frac{\partial \Phi}{\partial\left(\frac{\partial U}{\partial y_{i}}\right)}\right)+\frac{1}{\Delta} \sum_{i s} \frac{\partial^{2}}{\partial y_{i} \partial y_{s}}\left(\Delta \frac{\partial^{2} \Phi}{\partial\left(\frac{\partial^{2} U}{\partial y_{i} \partial y_{s}}\right)}\right)-\cdots
$$

ove $\Delta$ rappresenta il determinante funzionale:

$$
\frac{\partial\left(x_{1}, x_{2}, \ldots x_{n}\right)}{\partial\left(y_{1}, y_{2}, \ldots, y_{n}\right)}
$$

$\Gamma$ è la trasformata della $G$, ossia si ha identicamente:

$$
G=\Gamma,
$$

in virtù delle formule che legano le $x$ alle $y$.

La eguaglianza precedente si dimostra assai facilmente coi principii del calcolo delle variazioni. Difatti, fissato un campo finito di integrazione nello spazio ad $n$ dimensioni delle variabili $x$, per la formula di trasformazione 
degli integrali multipli si ha:

$$
\int^{(n)} F d x_{1} \ldots d x_{n}=\int^{(n)} \Phi \Delta d y_{1} \ldots d y_{n}
$$

ove il primo integrale è esteso al campo fissato, ed il secondo al campo corrispondente nello spazio delle variabili $y$. Se ora calcoliamo le variazioni di questi due integrali quando $U$ diviene $U+\delta U$, e riduciamo coi procedimenti soliti le espressioni sotto i segni d'integrazione ad essere funzioni lineari di $\delta U$, troviamo, poichè le variazioni al contorno del campo sono indipendenti da quelle all' interno,

$$
\int^{(n)} \delta U G d x_{1} \ldots d x_{n}=\int^{(n)} \partial U \Delta \mathrm{V} d y_{1} \ldots d y_{n} .
$$

Da questa equazione osservando che nel primo integrale, se si mutano le variabili $x$ nelle $y$, l'elemento $d x_{1} \ldots d x_{n}$ si muta in $\Delta d y_{1} \ldots d y_{n}$, e che il campo d'integrazione è arbitrario, si ottiene subito la (1), ammessa naturalmente la continuità delle espressioni $G, \Gamma$.

È questa la dimostrazione di J $\mathrm{J}_{\mathrm{AcOB}}$. Se per $F$ prendiamo la espressione:

$$
F=\sum_{i h} a_{i h} \frac{\partial U}{\partial x_{i}} \frac{\partial V}{\partial x_{h}}=\Delta_{\mathrm{s}}[U, V]
$$

ove $U, V$ sono due funzioni arbitrarie, e le $a_{i h}$ sono costanti, le sua trasformata sara:

$$
\Phi=\sum_{i h} b_{i h} \frac{\partial U}{\partial y_{i}} \frac{\partial V}{\partial y_{h}},
$$

ove le $b_{i h}$ sono funzioni delle $y$, e troviamo:

$$
G=-\sum_{i h} a_{i h} \frac{\partial^{2} U}{\partial x_{i} \partial x_{h}}=-\Delta_{2}[U]
$$

considerando $F, \Phi$ come dipendenti dalla $V$; e quindi:

$$
\Gamma=-\frac{1}{\Delta} \sum_{i \hbar h} \frac{\partial}{\partial y_{h}}\left(\Delta b_{i h} \frac{\partial U}{\partial y_{i}}\right) \text {. }
$$

Le formule di trasformazione delle $\frac{\partial U}{\partial x_{i}}, \frac{\partial V}{\partial x_{h}}$ nelle $\frac{\partial U}{\partial y_{i}}, \frac{\partial V}{\partial y_{h}}$ sono lineari, quindi la espressione $\Delta_{1}[U, V]$ considerata come forma bilineare di questi due sistemi di variabili ammette come invariante il discriminante:

$$
a=\mathbf{\Sigma} \pm a_{11} \ldots a_{n n}
$$


rispetto alle trasformazioni lineari il cui modulo è il determinante funzionale:

$$
\nabla=\frac{\partial\left(y_{1}, y_{2}, \ldots y_{n}\right)}{\partial\left(x_{1}, x_{2}, \ldots x_{n}\right)}
$$

indicando con $b$ il discriminante della forma trasformata:

$$
b=\mathbf{\Sigma} \pm b_{11} \ldots b_{n n}
$$

avremo quindi, poichè il discriminante è d'indice 2 ,

ossia:

$$
b=\nabla^{2} a,
$$

$$
\frac{1}{\Delta}=\sqrt{\frac{b}{a}} \text {. }
$$

Sostituendo questo valore di $\Delta$ nella (2) il secondo membro viene ad essere formato unicamente colle $b_{i n}$, le loro derivate prime e le derivate della $U$. La trasformazione della espressione $\Delta_{2}[U]$ richiede quindi unicamente la conoscenza dei coefficienti $b_{i n}$.

Ciò posto, la maggiore generalità del principio di $\mathrm{J}_{\mathrm{AC} O \mathrm{Br}}$, a cui abbiamo accennato, fa subito pensare se, mediante di esso, non sia possibile in generale ridurre la trasformazione delle espressioni lineari omogenee a coefficienti costanti di ordine qualunque $\nu$ :

$$
\Delta_{y}[U]=\underset{i_{1} i_{2} \ldots i_{y}}{\mathbf{y}} \alpha_{i_{1} i_{2} \ldots i_{2}} \frac{\partial^{\nu} U}{\partial x_{i_{1}} \partial x_{i_{2}} \ldots \partial x_{i_{y}}},
$$

alla trasformazione delle espressioni del $1 .^{\circ}$ ordine:

$$
\Delta_{1}\left[U_{1}, U_{2}, \ldots U_{y}\right]={\underset{i_{1}}{i_{3} \ldots i_{y}}}_{i_{i_{1}} i_{2} \ldots i_{y}} \frac{\partial U_{1}}{\partial x_{i_{1}}} \frac{\partial U_{2}}{\partial x_{i_{3}}} \cdots \frac{\partial U_{v}}{\partial x_{i_{y}}},
$$

come avviene nel caso di $y=2$.

Ora è facile persuadersi che questa riduzione mediante il principio di $J_{A C O B I}$ non è possibile appena si oltrepassano le espressioni del $2 .^{\circ}$ ordine; soltanto si può ridurre la trasformazione a quella di certe espressioni di ordine inferiore a $\nu$, ma maggiore di 1 , come ora vedremo. Intanto una delle ragioni che rendono impossibile la riduzione accennata sta nel fatto che ad espressioni come la $\Delta_{1}\left[U_{1}, U_{2}, \ldots U_{n}\right]$ il principio di $J_{A C O B I}$ non può essere applicato due volte. Se infatti prendiamo per $F$ questa espressione e la consideriamo come dipendente dalla funzione $U_{1}$, per la $G$ corrispondente, che possiamo indicare con $G_{1}$, troviamo:

$$
G_{1}=-i_{i_{1}} \mathbf{\Sigma}_{i_{2} \ldots i_{\psi}} a_{i_{1} i_{2} \ldots i_{y}} \frac{\partial}{\partial x_{i_{1}}}\left(\frac{\partial U_{2}}{\partial x_{i_{2}}} \cdots \frac{\partial U_{\nu}}{\partial i_{i_{\nu}}}\right),
$$


per cui il principio di J J diamo per $F$ la $G_{1}$, e la consideriamo come dipendente da $U_{2}$, osservando che si può scrivere:

$$
G_{1}=-\underset{i_{1}}{\mathbf{\Sigma} i_{3} \ldots i_{y}} a_{i_{1} i_{2} \ldots i_{y}}\left(\frac{\partial^{2} U_{2}}{\partial x_{i_{1}} \partial x_{i_{2}}} \frac{\partial U_{3}}{\partial x_{i_{3}}} \cdots \frac{\partial U_{\nu}}{\partial x_{i_{y}}}+\frac{\partial U_{2}}{\partial x_{i_{2}}} \frac{\partial}{\partial x_{i_{1}}}\left(\frac{\partial U_{3}}{\partial x_{i_{3}}} \cdots \frac{\partial U_{y}}{\partial x_{i_{\nu}}}\right)\right\},
$$

troviamo per la $G$ corrispondente:

$$
G_{2}=-\sum_{i_{2}} \frac{\partial}{\partial x i_{2}} \frac{\partial G_{1}}{\partial\left(\frac{\partial U_{2}}{\partial x i_{2}}\right)}+\sum_{i_{1} i_{2}}^{\mathbf{y}} \frac{\partial^{2}}{\partial x_{i_{1}} \partial x i_{2}} \frac{\partial G_{1}}{\partial\left(\frac{\partial^{2} U}{\partial x_{i_{1}} \partial x i_{2}}\right)}=0
$$

quindi è zero anche la $\Gamma$ corrispondente.

Vediamo ora quali sono invece le riduzioni che il principio di $J_{A C O B I}$ permette di fare. La espressione $\Delta_{\nu}[U]$ può essere scritta:

$$
\Delta_{\nu}[U]=\sum_{i_{1} i_{2} \ldots i_{\lambda}}^{\mathbf{\Sigma}} \frac{\partial^{\lambda}}{\partial x_{i_{1}} \partial x_{i_{2}} \ldots \partial x_{i_{\lambda}}} \sum_{i_{\lambda+1} \ldots i_{\nu}}^{\mathbf{y}} a_{i_{1} i_{2} \ldots i_{\nu}} \frac{\partial^{\nu-\lambda} U}{\partial x_{i_{\lambda+1}} \ldots \partial x_{i_{\nu}}},
$$

e questa espressione può considerarsi come la $G$ corrispondente alla $F$ definita da:

$$
F=(-1)^{\lambda} \underset{i_{1} i_{3} \ldots i_{\nu}}{\mathbf{y}} a_{i_{1} i_{2} \ldots i_{\nu}} \frac{\partial^{\lambda} V}{\partial x_{i_{1}} \ldots \partial x_{i_{\lambda}}} \frac{\partial^{\mu-\lambda} U}{\partial x_{i_{\lambda+1}} \ldots \partial x_{i_{\nu}}},
$$

e considerata come dipendente da $V$. Quindi se $\nu$ è pari possiamo prendere:

$$
\lambda=\nu-\lambda=\frac{\nu}{2},
$$

e se $\nu$ è dispari:

$$
\lambda=\frac{\nu+1}{2} \quad \nu-\lambda=\frac{\nu-1}{2} .
$$

Nel primo caso la trasformazione si riduce a quella di una espressione di ordine $\frac{\nu}{2}$, nel secondo di ordine $\frac{\nu+1}{2}$. In ogni caso poi potremo sempre prendere per $\lambda$ uno qualunque dei valori $1,2, \ldots, \nu-1$.

Visto adunque che il principio di $J_{A C O B I}$ non basta per eseguire la riduzione indicata, cerchiamo per altra via di decidere se tale riduzione non sia effettivamente possibile in modo assoluto, oppure se la difficoltà incontrata si debba al metodo, che abbiamo seguito. Osserviamo che nella trasformata (2) della espressione $\Delta_{2}[U]$ fra i coefficienti delle derivate del $10^{\circ}$ ordine e quelli delle derivate del $2 .^{\circ}$ esistono delle relazioni, per cui i primi si possono espri- 
mere in funzione dei secondi, $\theta$ che la stessa riduzione alla forma (2) si potrebbe ottenere qualora si trovassero per altra via le relazioni che esistono fra le due serie di coefficienti. Noi ci proporremo quindi la ricerca diretta delle relazioni fra i coefficienti di una equazione lineare che sia la trasformata di una equazione lineare, omogenea, a coefficienti costanti. Alcune di queste relazioni intanto ci sono fornite dalle riduzioni che si deducono dal principio di $\mathrm{J}_{A \mathrm{COBI}}$; noi però col metodo, che ora indicheremo, arriveremo a trovare anche queste relazioni indipendentemente dal principio stesso.

\section{§ 2. Proprietà delle funzioni $H_{q r, p}, K_{q r, p}$.}

Prima di indicare il metodo generale che seguiremo per eseguire la trasformazione, tenendo conto delle relazioni fra i coefficienti, è necessario stabilire alcune proprietà di certe espressioni, che ora definiremo e che godono di una importanza notevolissima in tutta la teoria della trasformazione.

Dalle formule ordinarie per il cambiamento delle variabili indipendenti si ha:

$$
\left.\begin{array}{ll}
\sum_{i} \frac{\partial y_{p}}{\partial x_{i}} \frac{\partial x_{i}}{\partial y_{q}}=\varepsilon_{p q} & (p, q=1,2, \ldots n) \\
\sum_{i h} \frac{\partial^{2} y_{p}}{\partial x_{i} \partial x_{h}} \frac{\partial x_{i}}{\partial y_{q}} \frac{\partial x_{h}}{\partial y_{r}}+\sum_{i} \frac{\partial^{2} x_{i}}{\partial y_{q} \partial y_{r}} \frac{\partial y_{p}}{\partial x_{i}}=0 & (p, q, r=1,2, \ldots n),
\end{array}\right\}
$$

dove $\varepsilon_{p q}$ è zero quando $p$ è differente da $q$, ed 1 quando $p=q$. Altre formule analoghe alle precedenti si hanno scambiando le $x$ colle $y$. Noi potremo ora porre:

$$
H_{q r, p}=\sum_{i h} \frac{\partial^{2} y_{p}}{\partial x_{i} \partial x_{h}} \frac{\partial x_{i}}{\partial y_{q}} \frac{\partial x_{h}}{\partial y_{r}}=-\sum_{i} \frac{\partial^{2} x_{i}}{\partial y_{q} \partial y_{r}} \frac{\partial y_{p}}{\partial x_{i}},
$$

e derivando queste due espressioni equivalenti di $H_{q r, p}$ troviamo:

$$
\begin{aligned}
& \frac{\partial H_{q r, p}}{\partial y_{s}}=\underset{i \hbar h}{\mathbf{y}} \frac{\partial^{3} y_{p}}{\partial x_{i} \partial x_{h} \partial x_{l}} \frac{\partial x_{i}}{\partial y_{q}} \frac{\partial x_{h}}{\partial y_{r}} \frac{\partial x_{l}}{\partial y_{s}}+ \\
& +\sum_{i \hbar} \frac{\partial^{2} y_{p}}{\partial x_{i} \partial x_{h}}\left(\frac{\partial^{2} x_{i}}{\partial y_{q} \partial y_{s}} \frac{\partial x_{h}}{\partial y_{r}}+\frac{\partial x_{i}}{\partial y_{q}} \frac{\partial^{2} x_{h}}{\partial y_{r} \partial y_{s}}\right) \\
& \frac{\partial H_{q r, p}}{\partial y_{s}}=-\sum_{i} \frac{\partial^{3} x_{i}}{\partial y_{q} \partial y_{r} \partial y_{s}} \frac{\partial y_{p}}{\partial x_{i}}-\sum_{i \hbar} \frac{\partial^{2} x_{i}}{\partial y_{q} \partial y_{r}} \frac{\partial^{2} y_{\dot{p}}}{\partial x_{i} \partial x_{h}} \frac{\partial x_{h}}{\partial y_{s}} \text {. }
\end{aligned}
$$


Noi porremo:

$$
\begin{aligned}
& H_{q r s, p}=\sum_{i h l} \frac{\partial^{3} y_{p}}{\partial x_{i} \partial x_{h} \partial x_{l}} \frac{\partial x_{i}}{\partial y_{q}} \frac{\partial x_{h}}{\partial y_{r}} \frac{\partial x_{l}}{\partial y_{s}} \\
& K_{q r s, p}=\sum_{i} \frac{\partial^{3} x_{i}}{\partial y_{q} \partial y_{r} \partial y_{s}} \frac{\partial y_{p}}{\partial x_{i}},
\end{aligned}
$$

e poichè si ha:

$$
\begin{aligned}
\sum_{i \hbar h} \frac{\partial^{2} y_{p}}{\partial x_{i} \partial x_{h}} \frac{\partial^{2} x_{i}}{\partial y_{q} \partial y_{s}} \frac{\partial x_{h}}{\partial y_{r}}=\sum_{i h j}^{\mathbf{y}} \frac{\partial^{2} y_{p}}{\partial x_{i} \partial x_{h}} \frac{\partial^{2} x_{j}}{\partial y_{q} \partial y_{s}} \frac{\partial x_{h}}{\partial y_{r}} \underset{k}{\mathbf{y}} \frac{\partial x_{i}}{\partial y_{k}} \frac{\partial y_{k}}{\partial x_{j}}= \\
=-\sum_{k} H_{r k, p} H_{q s, k} \\
\begin{aligned}
\sum_{i h} \frac{\partial^{2} x_{i}}{\partial y_{q} \partial y_{r}} \frac{\partial^{2} y_{p}}{\partial x_{i} \partial x_{h}} \frac{\partial x_{h}}{\partial y_{s}}=\sum_{i h_{j}} \frac{\partial^{2} x_{j}}{\partial y_{q} \partial y_{r}} \frac{\partial^{2} y_{p}}{\partial x_{i} \partial x_{h}} \frac{\partial x_{h}}{\partial y_{s}} \underset{\frac{1}{k}}{\mathbf{y}} \frac{\partial x_{i}}{\partial y_{k}} \frac{\partial y_{k}}{\partial x_{j}}= \\
=-\sum_{k} H_{s k, p} H_{q r, k},
\end{aligned}
\end{aligned}
$$

troviamo:

$$
\left.\begin{array}{l}
\frac{\partial H_{q r, p}}{\partial y_{s}}=H_{q r s, p}-\sum_{\vec{k}}\left(H_{r k, p} H_{q s, k}+H_{q k, p} H_{r s, k}\right) \\
\frac{\partial K_{q r, p}}{\partial y_{s}}=K_{q r s, p}-\sum_{\vec{k}} K_{s k, p} K_{q r, k},
\end{array}\right\}
$$

ove, per simmetria, abbiamo introdotto anche il simbolo:

$$
K_{q r, p}=\sum_{i} \frac{\partial^{2} x_{i}}{\partial y_{q} \partial y_{r}} \frac{\partial y_{p}}{\partial x_{i}}
$$

Da queste relazioni si ricava la seguente:

$$
-K_{q r s, p}=H_{q r s, p}-\sum_{k}\left(H_{q k, p} H_{r s, k}+H_{r k, p} H_{s q, k}+H_{s k, p} H_{q r, k}\right),
$$

la quale non è altro che quella che risulta dalla seconda delle (3) derivando rispetto ad $y_{s}$.

Dalle (4) si possono ricavare le $H_{q r s, p}, K_{q r s, p}$ in funzione delle $H_{q r, p}$ e delle loro derivate prime; questa proprietà non è che un caso particolare di una assai più generale. Poniamo infatti:

$$
H_{q_{1} q_{2} \ldots q_{\mu}, p}=\sum_{i_{1} i_{2} \ldots i_{\mu}} \frac{\partial^{\mu} y_{p}}{\partial x_{i_{1}} \partial x_{i_{2}} \ldots \partial x_{i_{\mu}}} \frac{\partial x_{i_{1}}}{\partial y_{q_{1}}} \frac{\partial x_{i_{2}}}{\partial y_{q_{2}}} \cdots \frac{\partial x_{i_{\mu}}}{\partial y_{q_{\mu}}},
$$

e deriviamo rispetto ad $y_{q_{\mu+1}}$. Con un artificio analogo a quello che ci ha 
servito a stabilire le (4) troviamo subito:

$$
\left.\begin{array}{l}
H_{q_{1} q_{2} \ldots q_{\mu+1}, p}=\frac{\partial H_{q_{1} q_{2} \ldots q_{\mu}, p}}{\partial y q_{\mu+1}}+ \\
\quad+\sum_{k}\left(H_{q_{1} q_{\mu+1}, p} H_{q_{2} q_{3} \ldots q_{\mu} k, p}+\cdots+H_{q_{\mu} q_{\mu+1}, p} H_{q_{1} q_{2} \ldots q_{\mu-1} k, p}\right) .
\end{array}\right\}
$$

Questa formula dimostra che, se $H_{q_{1} q_{2} \ldots q_{\mu}, p}$ è esprimibile in funzione delle $H_{q r, p}$ e delle loro derivate, anche $H_{q_{1} q_{2} . ., q_{\mu_{+1}}, p}$ gode della stessa proprietà; da ciò, e dalla prima delle (4), segue che tutte le $H_{q_{1} q_{2} \ldots q_{\mu}, p}$ con qualsiasi numero di indici sono funzioni razionali intere delle $H_{q r, p}$ e delle loro derivate rispetto alle $y$.

Il primo membro della (5) è simmetrico rispetto ai $\mu+1$ indici $q_{1}, q_{2}, \ldots$ $q_{\mu+1}$, non così il secondo; questa formula ci dà quindi $\mu+1$ espressioni equivalenti di $H_{q_{1} q_{2} \ldots q_{\mu+1}, p}$.

Similmente se si pone:

$$
K_{q_{1} q_{2} \ldots q_{\mu}, p}=\sum_{i} \frac{\partial^{\mu} x_{i}}{\partial y_{q_{1}} \partial y_{q_{2}} \ldots \partial y_{q_{\mu}}} \frac{\partial y_{p}}{\partial x_{i}}
$$

si trova:

$$
K_{q_{1} q_{2} \ldots q_{\mu+1}, p}=\frac{\partial K_{\left.q_{1} q_{2, \ldots} q_{\mu, p}\right)}}{\partial y_{q_{\mu+1}}}+\sum_{k} K_{q_{\mu+1} k, p} K_{q_{1} q_{2} \ldots q_{\mu}, k} .
$$

Questa formula, insieme alla seconda delle (4), dimostra per le $K_{q_{1} q_{2} \ldots q_{\mu}, p}$ una proprietà analoga a quella dimostrata per le $H_{q_{1} q_{2} \ldots q_{\mu}, p}$.

Queste proprietà sono importanti nella teoria della trasformazione, poichè da esse risulta che una volta calcolate le $H_{q r, p}$ in funzione delle nuove variabili indipendenti, tutte le altre quantità $H$ e $K$ con un numero qualsiasi di indici, si potranno avere dalle prime senza ricorrere alle formule, che legano le antiche variabili alle nuove.

Indicando ancora con $\Delta$ il determinante funzionale delle $x$ rispetto alle $y$, come nel $\S 1$, si ha:

e quindi:

$$
\frac{\partial y h}{\partial x_{i}}=\frac{1}{\Delta} \frac{\partial \Delta}{\partial\left(\frac{\partial x_{i}}{\partial y_{h}}\right)}
$$

$$
\frac{\partial \Delta}{\partial y_{s}}=\sum_{i h} \frac{\partial \Delta}{\partial\left(\frac{\partial}{\partial} \frac{x_{i}}{y_{h}}\right)} \frac{\partial^{2} x_{i}}{\partial y_{h} \partial y_{s}}=\Delta \sum_{i h} \frac{\partial^{2} x_{i}}{\partial y_{h} \partial y_{s}} \frac{\partial y_{h}}{\partial x_{i}},
$$


ossia :

$$
\frac{1}{\Delta} \frac{\partial \Delta}{\partial y_{s}}=-\frac{1}{\nabla} \frac{\partial \nabla}{\partial y_{s}}=-\sum_{h} H_{s h, h}
$$

formula di cui faremo frequente uso in seguito.

\section{§3. Metodo generale per la trasformazione.}

Riprendiamo l'espressione generale che dobbiamo trasformare:

$$
\Delta_{\nu}[U]=\sum_{i_{1} i_{2} \ldots i_{\nu}} a_{i_{1} i_{2} \ldots i_{\nu}} \frac{\partial^{\nu} U}{\partial x_{i_{1}} \partial x_{i_{2}} \ldots \partial x_{i_{\nu}}} .
$$

Sostituendo alle variabili $x$ le $y$, la trasformata sarà della forma seguente:

$$
\Delta_{\nu}[U]=\sum_{l_{1}} \sum_{2 . ., l_{\nu}} \sum_{\lambda=1}^{\nu} b_{l_{1}} l_{2} \ldots l_{\lambda} \frac{\partial^{\lambda} U}{\partial y l_{1} \partial y l_{2} \ldots \partial y l_{\lambda}},
$$

ove i coefficienti $b_{l_{1}} l_{2} \ldots l_{\lambda}$ sono funzioni delle $y$, la cui forma generale è:

$$
\begin{aligned}
& b_{l_{1} l_{2} \ldots l_{\nu}}=\sum_{i_{1} i_{3} \ldots i_{\nu}} a_{i_{1} i_{2} \ldots i_{\nu}} \frac{\partial y l_{1}}{\partial x i_{1}} \cdots \frac{\partial y l_{\nu}}{\partial x_{i_{\nu}}} \\
& b_{l_{1} l_{2} \ldots l_{\nu-1}}=\sum_{i_{1} i_{2} \ldots i_{\nu}} a_{i_{1} i_{2} \ldots i_{\nu}}\left(\frac{\partial^{2} y l_{1}}{\partial x i_{1} \partial x i_{\nu}} \frac{\partial y l_{2}}{\partial x_{i_{9}}} \cdots \frac{\partial y_{l_{\nu-1}}}{\partial x i_{y_{-1}}}+\cdots\right) \\
& b_{l_{1}}=\sum_{i_{1} i_{2} \ldots i_{\nu}}^{\mathbf{\Sigma}} a_{i_{1} i_{2} \ldots i_{\nu}} \frac{\partial^{y} y l_{1}}{\partial x_{i_{1}} \partial x_{i_{2}} \ldots \partial x i_{\nu}} .
\end{aligned}
$$

In ciascuno dei termini, che compaiono sotto $\mathrm{i}$ segni sommatori nei secondi membri, è costante ed uguale a y il numero delle derivazioni che vi si devono eseguire; quindi possiamo dire che $i$ coefficienti $b$ sono funzioni lineari a coefficienti numerici di tutte le espressioni che si possono formare dalla

$$
\begin{aligned}
& \Delta\left[y_{l_{1}}\left|y_{l_{2}}\right| \ldots \mid y_{l_{r}}\right]=
\end{aligned}
$$

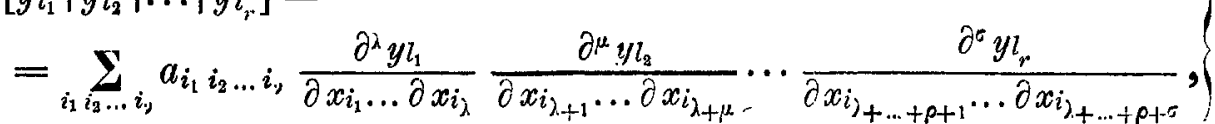

attribuendo a $\lambda, \mu, \ldots \rho, \sigma$ tutti $\mathrm{i}$ possibjli valori interi positivi o nulli, che soddisfanno alla equazione:

$$
\lambda+\mu+\cdots+\rho+\sigma=\nu .
$$


Ora osserviamo che, ricorrendo al solito artificio, possiamo scrivere nel secondo membro della (10) al posto di

l'espressione:

$$
a_{i_{1} i_{2} \ldots i_{\nu}} \frac{\partial^{\lambda} y l_{1}}{\partial x_{i_{1}} \partial x_{i_{2} \ldots \partial i_{\lambda}}}
$$

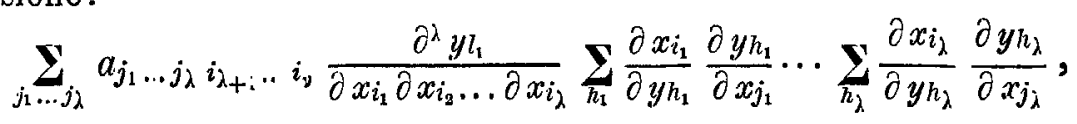

e quel secondo membro diverrà:

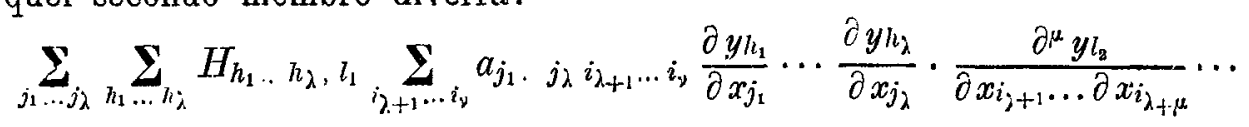

Eseguendo una riduzione analoga rispetto a tutte le altre derivate di ordine $\mu, \ldots \rho, \sigma$ troveremo al fine per la $\Delta\left[y_{l_{1}}\left|y l_{2}\right| \ldots \mid y l_{r}\right]$ la espressione:

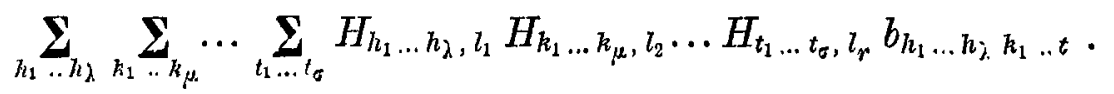

Quindi, ricordando la proprietà dimostrata nel paragrafo precedente per le funzioni $H_{q_{1} \ldots q_{\mu}, p}$, possiamo enunciare il seguente teorema:

I coefficienti $b_{l_{1}} . l_{\lambda}$ della trasformata della espressione (7) sono funzioni lineari delle $b_{l_{1}} \ldots l_{\nu}$ a ${ }_{\nu}$ indici, cioè dei coefficienti delle derivate di ordine $\nu$, e di certe espressioni razionali intere delle $H_{q r, p}$ e delle loro derivate rispetto alle $y$.

Da ciò segue, che se noi potremo esprimere le $H_{q r, p}$ in funzione delle $b_{l_{1} \ldots l}$, tutti $\mathrm{i}$ coefficienti della (8) si potranno esprimere in funzione unicamente di queste quantità, e delle loro derivate; ossia dei coefficienti (9) basterà conoscere, in funzione delle $y$, quelli dati dalla prima eguaglianza per poterne dedurre tutti i rimanenti.

Ora i coefficienti $b_{l_{1}} \ldots l_{1}$, sono anche quelli della trasformata di:

$$
\Delta_{1}\left[U_{1}, \ldots U_{v}\right]=\sum_{i_{1} \ldots i_{y}} a_{i_{1} \ldots i}, \frac{\partial U_{1}}{\partial x_{i_{1}}} \cdots \frac{\partial U_{v}}{\partial x_{i_{v}}},
$$

sicchè la quistione, che ci siamo proposti al $\S 1$, è ora ridotta a vedere se le funzioni $H_{q r, p}$ possono essere espresse mediante i coefficienti $b_{l_{1} \ldots l_{y}}$ e le loro derivate.

Ora osserviamo che derivando la prima delle (9) ed introducendo coll'artificio solito le $H_{g r, p}$ si trova:

$$
\frac{\partial b_{1} l_{2} \ldots l_{\nu}}{\partial y l}=\sum_{h}\left(H_{h l, l_{1}} b_{h l_{2} \ldots l_{\nu}}+\cdots+H_{h l, l_{\nu}} b_{l_{1} l_{2} \ldots l_{\nu-1} h}\right) .
$$


Queste identità sono in numero di

$$
M=n \cdot \frac{n(n+1) \cdots(n+\nu-1)}{1 \cdot 2 \cdots v},
$$

quindi da esse, in generale, si potranno ricavare le

$$
N=n \cdot \frac{n(n+1)}{1 \cdot 2},
$$

quantità $H_{q r, p}$, che vi compariscono linearmente, in funzione delle $b_{l_{1} l_{2} \ldots l_{y}} \mathrm{e}$ delle loro derivate prime.

Possiamo quindi dire: la riduzione della trasformazione della $\Delta_{\nu}[U]$ a quella della $\Delta_{1}\left[U_{1}, \ldots, U_{\Downarrow}\right]$ è effettuabile, compatibilmente colla possibilità di risolvere le equazioni lineari (11) rispetto alle $H_{q r, p}$.

Nella maggior parte dei casi però, invece delle (11), si possono considerare altre equazioni, la cui risoluzione è assai più semplice, poichè da esse si possono dedurre dei gruppi di $n$ equazioni, ciascuno dei quali contiene linearmente soltanto $n$ delle $H_{q r, p}$; la determinazione di queste funzioni si riduce quindi alla risoluzione successiva di questi sistemi di $n$ equazioni soltanto. Ciò avviene tutte le volte che la espressione $\Delta_{1}\left[U_{1}, \ldots, U_{v}\right]$ può essere considerata come un invariante assoluto di una forma differenziale di un grado qualsiasi $\rho$, maggiore di 1 . Sia infatti:

$$
\varphi=\sum_{r_{1} r_{2} \ldots r_{p}} \alpha_{r_{1} r_{2} \ldots r_{p}} d x_{r_{1}} d x_{r_{2}} \ldots d x_{r_{p}},
$$

questa forma, ove le a sono funzioni delle a. Sostituendo alle variabili $x$ le $y$, essa diverrà:

$$
\varphi=\sum_{s_{1} s_{2} \ldots s_{p}} \beta_{s_{1} s_{2} \ldots s_{p}} d y_{s_{1}} d y_{s_{2}} \ldots d y_{s_{p}},
$$

e, per ipotesi, le $\beta$ saranno formate colle $b$ in modo identico a quello con cui le a sono formate colle a. Arremo poi:

$$
\beta_{s_{1} s_{2} \ldots s_{\rho}}=\sum_{r_{1} r_{2} \ldots r_{\rho}} \alpha_{r_{1} r_{2} \ldots r_{\rho}} \frac{\partial x_{r_{1}}}{\partial y_{s_{1}}} \cdots \frac{\partial x_{r_{\rho}}}{\partial y_{s_{\rho}}}
$$

Derivando rispetto ad $y_{s}$ troviamo:

$$
\frac{\partial \beta_{s_{1} s_{2} \ldots s_{p}}}{\partial y_{s}}=\sum_{k}\left(K_{s s_{1}, k} \beta_{k s_{2} \ldots s_{p}}+\cdots+K_{s s_{\rho}, k} \beta_{s_{1} s_{1} \ldots s_{\rho-1} k}\right),
$$

equazioni che sono analoghe alle (11). Noi porremo, per semplicità,

$$
\sum_{k} K_{s s_{i}, k} \beta_{s_{1} \ldots s_{i-1}} k s_{i+1} \ldots s_{p}=\left(s s_{i}\right)\left[s_{1} \ldots s_{i-1} s_{i+1} \ldots s_{\rho}\right]
$$


e avremo dalla (11') le seguenti formule:

$$
\begin{aligned}
& \frac{\partial \beta_{t s_{2} \ldots s_{\rho}}}{\partial y_{s_{1}}}=\left(t s_{1}\right)\left[s_{2} \ldots s_{\rho}\right]+\left(s_{2} s_{1}\right)\left[t s_{3} \ldots s_{\rho}\right]+\cdots+\left(s_{\rho} s_{1}\right)\left[t s_{2} \ldots s_{\rho-1}\right] \\
& \frac{\partial \beta_{s_{1} t s_{6} \ldots s_{p}}}{\partial y_{s_{2}}}=\left(s_{1} s_{2}\right)\left[t s_{3} \ldots s_{p}\right]+\left(t s_{2}\right)\left[s_{1} s_{3} \ldots s_{p}\right]+\cdots+\left(s_{p} s_{2}\right)\left[s_{1} t s_{3} \ldots s_{p-1}\right]
\end{aligned}
$$

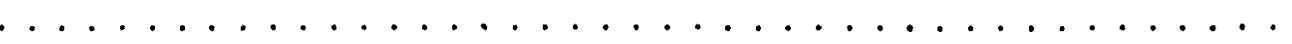

$$
\begin{aligned}
& \frac{\partial \beta_{s_{1} \ldots s_{p-1} t}}{\partial y_{s_{p}}}=\left(s_{1} s_{p}\right)\left[s_{2} \ldots s_{p-1} t\right]+\left(s_{2} s_{p}\right)\left[s_{1} s_{3} \ldots s_{p-1} t\right]+\cdots+\left(t s_{p}\right)\left[s_{1} \ldots s_{p-1}\right] \\
& \frac{\partial \beta_{s_{1} s_{2} \ldots s_{p}}}{\partial y_{t}}=\left(s_{1} t\right)\left[s_{2} \ldots s_{p}\right]+\left(s_{2} t\right)\left[s_{1} s_{3} \ldots s_{p}\right]+\cdots+\left(s_{r} t\right)\left[s_{1} \ldots s_{p-1}\right]
\end{aligned}
$$

da queste equazioni ponendo:

si ottiene:

$$
\frac{1}{2}\left(\frac{\partial \beta_{t s_{2} \ldots s_{p}}}{\partial y_{s_{1}}}+\cdots+\frac{\partial \beta_{s_{1}, . . s_{p-1} t}}{\partial y_{s_{p}}}-\frac{\partial \beta_{s_{1} \ldots s_{p}}}{\partial y_{t}}\right)=\beta_{s_{1} \ldots s_{p}, t}
$$

$$
\beta_{s_{1} \ldots s_{0}, t}=\sum_{(2 h)}\left(s_{i} s_{h}\right)\left[t s_{1} \ldots s_{i-1} s_{i+1} \ldots s_{h-1} s_{h+1} \ldots s_{p}\right],
$$

ove il simbolo $(i h)$, posto sotto il segno sommatorio, indica che la somma rispetto agli indici $i, h$ va estesa alle $\frac{n(n-1)}{1 \cdot 2}$ combinazioni due a due dei numeri $1,2, \ldots n$.

Delle (13) possiamo servirci per ricavare le $H_{q r . p}$; difatti facciamo dapprima $s_{1}=s_{2}=\cdots=s_{\rho}=s$ e sostituiamo alle parentesi del secondo membro le loro espressioni effettive. Arremo:

$$
\beta_{s s \ldots s, t}=-\frac{n(n-1)}{1 \cdot 2} \sum_{h} H_{s s, k} \beta_{k t s \ldots s} .
$$

Se ora attribuiamo a $t$ tutti $\mathrm{i}$ valori da 1 ad $n$, otterremo un sistema di $n$ equazioni lineari rispetto alle $n$ quantità $H_{s s, 1}, H_{s s, 2}, \ldots H_{s s, h}$. Quindi se indichiamo con $B_{k t, s}$ il complemento algebrico dell'elemento $\beta_{k t s s_{. . .} s}$ nel determinante:

$$
\left|\begin{array}{ccc}
\beta_{11 s \ldots s} & \beta_{12 s \ldots s} \ldots & \beta_{1 n s \ldots s} \\
\ldots \ldots & \ldots \ldots & \ldots \\
\beta_{n 1 s \ldots s} & \beta_{n 2 s} \ldots s . . & \beta_{n n s \ldots s}
\end{array}\right|
$$

diviso pel determinante stesso, avremo:

$$
H_{s s, p}=-\frac{1 \cdot 2}{n(n-1)} \sum_{t} \beta_{s \ldots s, t} B_{p t, s} .
$$


Otteniamo così, quando gli $n$ determinanti che si hanno dal precedente facendo $s=1,2, \ldots n$ sono differenti da zero, la espressione delle $H_{q r, p}$, i cui due primi indici sono uguali, in funzione delle $\beta$ e delle loro derivate prime, quindi anche delle $b$ e delle loro derivate.

Per ottenere ora anche le espressioni delle $H_{q r, p}$, i cui due primi indici sono disuguali, facciamo nella (13) $s_{1}=s_{2}=\cdots=s_{p-1}=s, s_{p}=r$; avremo:

$$
\beta_{s \ldots s r, t}=-\frac{(n-1)(n-2)}{1 \cdot 2} \sum_{i} H_{s, k, k} \beta_{t k r, \ldots s}-(n-1) \sum_{k} H_{s r, k} \beta_{t k s \ldots s},
$$

da cui ponendo per le $H_{s s, k}$ i valori dati dalle (15) si ha:

$$
(n-1) \sum_{l i} H_{s r, k} \beta_{t k s . . s}=-\beta_{s . . s r, t}+\frac{n-2}{n} \sum_{h k} \beta_{s \ldots s, h} B_{k h, s} \beta_{t k r s . . s},
$$

e risolvendo:

$$
\begin{aligned}
& H_{s r, p}=-\frac{1}{n-1} \sum_{t} \beta_{s \ldots s r, t} B_{p t, s}+ \\
& \quad+\frac{n-2}{n(n-1)} \sum_{h h t} \beta_{s_{\ldots, n}, h} B_{h k, s} B_{p t, s} \beta_{t h r s, s} .
\end{aligned}
$$

La condizione perchè questa formula abbia sempre un significato è, come per la (15), che siano differenti da zero gli $n$ determinanti (14). Osserviamo che nel solo caso in cui $\rho=2$, questi $n$ determinanti si riducono ad uno solo.

Notiamo infine che, se scriviamo tutte le $M$ equazioni (11) e formiamo la matrice dei coefficienti delle $H_{q r, p}$, la quale conterrà $N$ colonne ed $M$ linee, basterà che uno almeno dei determinanti di ordine $N$, che se ne possono dedurre sia differente da zero, perchè si possano risolvere le equazioni stesse rispetto alle $H_{q r, p}$. Per $\nu=2$ si ha $M=N$.

Riguardo a tutte queste condizioni si presenta una questione interessante: se cioè può avvenire che esistano trasformazioni per le quali esse sono soddisfatte, mentre per altre non lo sono; se così fosse, ne verrebbero delle limitazioni circa le trasformazioni per le quali è possibile determinare le $H_{q r, p}$ nel modo indicato. Non sembra però facile stabilire in proposito una discussione generale, ma conviene esaminare, caso per caso, il significato delle condizioni enunciate; noi ne redremo qualche esempio in seguito.

Riassumendo possiamo dire che per trovare la trasformata di una espressione $\Delta_{y}[U]$, in funzione soltanto dei coefficienti delle derivate di ordine $\nu$, noi dovremo dapprima trovare colle formule ordinarie come $\mathrm{i}$ coefficienti di tutte le derivate che compaiono nella trasformata siano composti colle espres- 
sioni $\Delta\left[y_{l_{1}}\left|y_{l_{2}}\right| \ldots \mid y l_{r}\right]$; poi costruire le corrispondenti espressioni $\left(10^{\prime}\right)$ e ridurle a dipendere soltanto dalle $H_{q r, p}$ colle formule del paragrafo precedente; infine sostituire per le $H_{q r, p}$ i valori (15) (15'), oppure i valori che si deducono dalle (11), secondo che esiste o no una forma differenziale covariante della $\Delta_{1}\left[U_{1}, \ldots, U_{v}\right]$.

Le espressioni $\Delta\left[U_{1}\left|U_{2}\right| \ldots \mid U_{r}\right]$ godono di una importanza notevole quando si studiano le equazioni:

$$
\Delta_{v}[U]=0,
$$

come in parte abbiamo già visto. Di esse ne esistono tante quante sono le partizioni possibili del numero $\nu$; i coefficienti delle loro trasformate sono della stessa forma (10) dei coefficienti della $\Delta_{v}[U]$; quindi la loro trasformazione si riduce, come per questa espressione, al calcolo dei coefficienti $b_{l \ldots l_{v}}$ a $\nu$ indici. Noi, per questa ragione, diremo che costituiscono il gruppo dei covarianti dell'equazione.

Applicheremo ora il metodo generale esposto ad alcuni casi speciali.

\section{§ 4. Equazioni di $2 .^{\circ}$ ordine.}

Per le equazioni del $2 .^{\circ}$ ordine le nostre formule conducono immediatamente ai risultati conosciuti. Si ha infatti per la trasformata della

$$
\Delta_{2}[U]=\sum_{i \hbar} a_{i h} \frac{\partial^{2} U}{\partial x_{i} \partial x_{h}}
$$

la espressione:

$$
\Delta_{2}[U]=\sum_{p q} b_{p q} \frac{\partial^{2} U}{\partial y_{p} \partial y_{q}}+\sum_{p} b_{p} \frac{\partial U}{\partial y_{p}}
$$

ove:

$$
b_{p q}=\Delta_{1}\left[y_{p}, y_{q}\right] \quad b_{p}=\Delta_{\varepsilon}\left[y_{p}\right] .
$$

Le formule $\left(10^{\prime}\right)$ ci dànno:

$$
b_{p}=\sum_{r s} H_{r s, p} b_{r s}
$$

e le (11):

$$
\frac{\partial b_{p q}}{\partial y_{r}}=\sum_{h}\left(H_{h r, p} b_{h q}+H_{h r, q} b_{h p}\right)
$$

relazioni, la cui verificazione è inoltre semplicissima. Se in quest'ultima fac- 
ciamo $r=q$ e sommiamo rispetto a $q$, ricordando la (6) troviamo:

ossia :

$$
\sum_{q} \frac{\partial b_{p q}}{\partial y_{q}}=\sum_{h q} H_{h q, p} b_{h q}-\frac{1}{\Delta} \sum_{h} \frac{\partial \Delta}{\partial y_{h}} b_{h p},
$$

$$
b_{p}=\frac{1}{\Delta} \sum_{q} \frac{\partial\left(\Delta b_{p q}\right)}{\partial y_{q}}
$$

Sostituendo questa espressione nella $(\alpha)$ troviamo:

$$
\Delta_{2}[U]=\frac{1}{\Delta} \sum_{p q} \frac{\partial}{\partial y_{q}}\left(\Delta b_{p q} \frac{\partial U}{\partial y_{p}}\right)
$$

che è la formula a cui si arriva col principio di $\mathrm{J}_{\mathrm{ACOBI}}$; in essa poi, come abbiamo visto nel $\S 1$, al posto di $\Delta$ si può sostituire $\frac{1}{\sqrt{b}}$, ove $b=\mathbf{\Sigma} \pm b_{11} \ldots b_{n n}$.

La espressione:

$$
\Delta_{1}[U, V]=\sum_{i h} a_{i h} \frac{\partial U}{\partial x_{i}} \frac{\partial V}{\partial x_{h}},
$$

è in questo caso il solo covariante dell'equazione. Formiamo ora le due forme differenziali:

$$
\begin{aligned}
d U d V & =\sum_{i h} \frac{\partial U}{\partial x_{i}} \frac{\partial V}{\partial x_{h}} d x_{i} d x_{h} \\
\varphi & =\sum_{i h} \alpha_{i h} d x_{i} d x_{h},
\end{aligned}
$$

ove, posto $a=\mathbf{\Sigma} \pm a_{11} \ldots a_{n}, \alpha=\mathbf{\Sigma} \pm \alpha_{11} \ldots \alpha_{n n}$, si ha:

$$
\alpha_{i h}=\frac{1}{a} \frac{\partial a}{\partial a_{i h}} \text { e quindi } \quad a_{i h}==\frac{1}{\alpha} \frac{\partial \alpha}{\partial \alpha_{i h}},
$$

considerando come distinti $a_{i h}$ e $a_{h i}$, $\alpha_{i h}$ e $\alpha_{h i}$. Queste forme, considerate come forme algebriche dei differenziali, hanno l'invariante simultaneo assoluto:

$$
\frac{1}{\alpha} \sum_{i \hbar} \frac{\partial \alpha}{\partial \alpha_{i h}} \frac{\partial U}{\partial x_{i}} \frac{\partial V}{\partial x_{h}}=\sum_{i \hbar} a_{i \hbar} \frac{\partial U}{\partial x_{i}} \frac{\partial V}{\partial x_{h}}
$$

e quindi $\Delta_{1}[U, V]$ può considerarsi come un invariante di $\varphi$.

La trasformata di $\varphi$ sia:

$$
\varphi=\sum_{r s} \beta_{r s} d y_{r} d y_{s}
$$

ove le $\beta_{r s}$ sono formate colle $b_{r \text { rs }}$ come le $\alpha_{i h}$ colle $a_{i h}$; le espressioni (12) 
sono in questo caso le seguenti:

e le (13) divengono:

$$
\beta_{r s, t}=\frac{1}{2}\left(\frac{\partial \beta_{t s}}{\partial y_{r}}+\frac{\partial \beta_{r t}}{\partial y_{s}}-\frac{\partial \beta_{r s}}{\partial y_{t}}\right),
$$

$$
\beta_{r s, t}=-\sum_{k}^{\mathbf{y}} H_{r s, k} \beta_{k t} .
$$

I determinanti (14) si riducono al solo discriminante:

$$
\beta=\mathbf{\Sigma} \pm \beta_{11} \ldots \beta_{n n},
$$

e le (15), (15') alle seguenti:

$$
H_{r s p}=-\mathbf{\Sigma}_{t} \beta_{r s, t} b_{p t},
$$

valevoli tanto per $r=s$, che per $r$ differente da $s$. Sostituendo questo valore di $H_{r s, p}$ nella $(\beta)$ si trova:

e, se poniamo:

$$
b_{p}=-\sum_{t r s} \beta_{r s, t} b_{p t} b_{r s}
$$

si ha:

$$
U_{t}=\sum_{p} b_{p t} \frac{\partial U}{\partial y_{t}},
$$

$$
\sum_{p} h_{p} \frac{\partial U}{\partial y_{p}}=-\sum_{r s t} \beta_{r s, t} b_{r s} U_{t} .
$$

Sostituendo nella $(\alpha)$ infine troviamo:

$$
\Delta_{2}[U]=\underset{p q q}{\mathbf{\Sigma}} b_{p q}\left(\frac{\partial^{2} U}{\partial y_{p} \partial y_{q}}-\underset{t}{\mathbf{Y}} \beta_{p q . t} U_{t}\right) .
$$

Questa forma dell'espressione $\Delta_{2}[U]$ è stata considerata dal prof. Riccr nelle ricerche, che abbiamo citato precedentemente.

Il discriminante $\beta$ deve essere differente da zero; ora, se questa condizione è soddisfatta per un sistema di variabili, lo è per qualsiasi altro, poichè si ha:

$$
\beta=\Delta^{2} \alpha=\frac{\Delta^{2}}{a},
$$

e quindi si vede che essa riguarda unicamente $\mathrm{i}$ coefficienti della espressione data $\Delta_{2}[U]$. 


\section{$\S 5$. Equazioni di $3 .^{\circ}$ ordine.}

Nel caso delle equazioni del $3 .^{\circ}$ ordine l'espressione:

si trasforma nella seguente:

$$
\Delta_{3}[U]=\sum_{i \hbar k_{k}} a_{i h k} \frac{\partial^{3} U}{\partial x_{i} \partial c_{h} \partial x_{k}},
$$

$$
\begin{array}{r}
\Delta_{3}[U]=\sum_{p w^{\prime}} \Delta_{1}\left[y_{p}, y_{q}, y_{p}\right] \frac{\hat{\partial}^{2} U}{\partial y_{p} \partial y_{q} \partial y_{r}}+3 \sum_{p q} \Delta_{2}\left[y_{p} \mid y_{q}\right] \frac{\hat{\partial}^{2} U}{\partial y_{p} \partial y_{q}}+ \\
+\sum_{p}^{\mathbf{y}} \Delta_{3}\left[y_{p}\right] \frac{\partial U}{\partial y_{p}},
\end{array}
$$

ove si ha:

$$
\begin{aligned}
\Delta_{1}\left[y_{p}, y_{q}, y_{r}\right] & =\sum_{i k h} a_{i h k} \frac{\partial y_{p}}{\partial x_{i}} \frac{\partial y_{q}}{\partial x_{h}} \frac{\partial y_{r}}{\partial x_{k}}=b_{p q r} \\
\Delta_{2}\left[y_{p} \mid y_{q}\right] & =\sum_{i h k} a_{i h k} \frac{\partial^{2} y_{p}}{\partial x_{i} \partial x_{h}} \frac{\partial y_{q}}{\partial x_{k}}=b_{p q} \\
\Delta_{3}\left[y_{p}\right] & =\sum_{i h l_{i}} a_{i h k} \frac{\partial^{3} y_{p}}{\partial x_{i} \partial x_{h} \partial x_{k}}=b_{p},
\end{aligned}
$$

e si può osservare che, se si introduce la notazione:

si ha anche:

$$
\Delta_{2}\left[y_{p}, y_{q}\right]=\Delta_{z}\left[y_{p} \mid y_{q}\right]+\Delta_{z}\left[y_{q} \mid y_{p}\right]
$$

$$
2 \sum_{y_{q}} \Delta_{q}\left[y_{p} \mid y_{q}\right] \frac{\partial^{2} U}{\partial y_{p} \partial y_{q}}=\sum_{p_{q}} \Delta_{z}\left[y_{p}, y_{q}\right] \frac{\partial^{2} U}{\partial y_{p} \partial y_{q}} .
$$

Le $\left(10^{\prime}\right)$ ci dànno:

quindi abbiamo:

$$
\begin{aligned}
& b_{p q}=\sum_{l m} H_{l m . p} b_{l n q} \\
& b_{p}=\sum_{l m t} H_{l m n, p} b_{l m n}
\end{aligned}
$$

$$
\Delta_{3}[U]=\sum_{p q r} b_{p q r}\left(\frac{\partial^{3} U}{\partial y_{p} \partial y_{q} \partial y_{r}}+3 \sum_{l} H_{p q, l} \frac{\partial^{2} U}{\partial y_{p} \partial y_{q}}+\sum_{l} H_{p q r, l} \frac{\partial U}{\partial y_{l}}\right) .
$$

Dalle (5) (11) poi si hanno le relazioni:

$$
\begin{aligned}
& H_{p q r, l}=\frac{\partial H_{p q . l}}{\partial y_{r}}+\underset{k}{2}\left(H_{p r, k} H_{q k, l}+H_{q r, k} H_{p k, l}\right) \\
& \frac{\partial b_{p \psi r}}{\partial y_{s}}=\frac{\mathbf{y}}{l}\left(H_{l s, p} b_{l q r}+H_{l s, q} b_{l r p}+H_{l s, r} b_{l p q}\right),
\end{aligned}
$$

per eliminare dalla $(\alpha)$ le $H_{p q r, l}, H_{p q, r}$, introducendo invece le derivate delle $b_{p q r}$. 
Se indichiamo con $B$ una opportuna potenza di un invariante qualsiasi della espressione $\Delta_{1}[U, V, W]$, considerata come forma algebrica delle derivate delle tre funzioni $U, V, W$, noi nella (6) al posto di $\Delta$ potremo sostituire $B$, analogamente a quanto abbiamo fatto nel caso delle equazioni di $2 .^{\circ}$ ordine (vedi $\S 1$ ), e avremo quindi:

$$
\underset{p}{\mathbf{v}} H_{l p p}=-\frac{1}{B} \frac{\partial B}{\partial y_{l}} \text {. }
$$

Ciò posto facendo nella $(\gamma) s=p$, e sommando rispetto a $p$ si trova:

$$
\frac{1}{B} \sum_{i} \frac{\partial}{\partial y l}\left(B b_{l_{q} r}\right)=b_{q r}+b_{r q} \text {. }
$$

Inoltre si ha:

$$
\frac{\mathbf{y}}{a} \frac{\partial b_{p q}}{\partial y_{q}}=\sum_{l_{q}}\left(\frac{\partial H_{l m, p}}{\partial y_{q}} b_{l m q}+H_{l m p} \frac{\partial b_{l n q}}{\partial y_{q}}\right)
$$

e sostituendo nel secondo membro $\mathrm{i}$ valori delle derivate che risultano dalle $(\beta)(\gamma)$, si trova:

ossia:

$$
\sum_{q} \frac{\partial b_{p q}}{\partial y_{q}}=b_{p}+\sum_{l m q^{k}}^{\mathbf{z}} H_{k q . q} H_{l n p} b_{k l m}
$$

$$
\frac{1}{B} \sum_{I} \frac{\partial}{\partial y_{q}}\left(B b_{p q}\right)=b_{p}
$$

Queste due relazioni $(\delta)(\varepsilon)$ sono quelle, a cui si arriverebbe col principio di $\mathrm{J}_{\Lambda \mathrm{COBI}}$; esse però, come si vede, non bastano per determinare le $b_{p q}, b_{p}$ in funzione delle $b_{p q r}$.

Supponiamo ora che sia $n=2$. In questo caso possiamo costruire una forma differenziale cubica, rispetto alla quale la espressione:

$$
\Delta_{1}[U, V, W]=\sum_{i h k}^{\mathbf{s}} a_{i h k} \frac{\partial U}{\partial x_{i}} \frac{\partial V}{\partial x_{h}} \frac{\partial W}{\partial x_{k}},
$$

sia un invariante. Difatti due forme binarie cubiche l'una di coefficienti $\alpha$, l'altra di coefficienti $\gamma$, hanno l'invariante simultaneo rappresentato simbolicamente da $(\alpha \gamma)^{3}$, che diviene un invariante assoluto, se noi lo dividiamo per la potenza $\frac{1}{2}$ del discriminante di una delle forme. Le due forme siano:

$$
\varphi=\sum_{i \pi k} \alpha_{i h k} d x_{i} d x_{h} d x_{k}=\alpha_{0} d x_{1}^{3}+3 \alpha_{1} d x_{1}^{2} d x_{2}+3 \alpha_{2} d x_{1} d x_{2}^{2}+\alpha_{3} d x_{2}^{3}
$$

$d U d V d W=\sum_{i h k} \frac{\partial U}{\partial x_{i}} \frac{\partial V}{\partial x_{h}} \frac{\partial W}{\partial x_{k}} d x_{i} d x_{h} d x_{k}$. 
Il discriminante di $\varphi \grave{e}:$

$$
R_{\alpha}=4\left(\alpha_{0} \alpha_{2}-\alpha_{1}^{2}\right)\left(\alpha_{1} \alpha_{3}-\alpha_{2}^{2}\right)-\left(\alpha_{0} \alpha_{3}-\alpha_{1} \alpha_{2}\right)^{2},
$$

c l'invariante simultaneo sovracennato $\dot{e}$ :

$$
\begin{aligned}
\frac{1}{R_{z}^{\frac{1}{2}}}\left\{\alpha_{0} \frac{\partial U}{\partial x_{2}} \frac{\partial V}{\partial x_{2}} \frac{\partial W}{\partial x_{2}}-\alpha_{1}\right. & \left(\frac{\partial U}{\partial x_{1}} \frac{\partial V}{\partial x_{2}} \frac{\partial W}{\partial x_{2}}+\cdots\right)+ \\
& \left.+\alpha_{2}\left(\frac{\partial U}{\partial x_{2}} \frac{\partial V}{\partial x_{1}} \frac{\partial W}{\partial x_{1}}+\cdots\right)-\alpha_{3} \frac{\partial U}{\partial x_{1}} \frac{\partial V}{\partial x_{1}} \frac{\partial W}{\partial x_{1}}\right\} .
\end{aligned}
$$

Se vogliamo che esso coincida con $\Delta_{1}[U, V, V]$ dovromo determinare le a in modo che sia:

$$
\frac{\alpha_{0}}{R_{\alpha}^{\frac{1}{2}}}=a_{3} \quad-\frac{\alpha_{1}}{R_{\varkappa}^{\frac{1}{3}}}=a_{2} \quad \frac{\alpha_{2}}{R_{\%}^{\frac{1}{2}}}=a_{1} \quad \frac{\alpha_{3}}{R_{\%}^{\frac{1}{2}}}=-a_{0},
$$

ove $a_{0}=a_{11}, \ldots a_{3}=a_{222} ;$ di qui, indicando con $R_{a}$ il discriminante della forma di coefficienti $a$, si ha:

$$
R_{\alpha}, R_{a}=1
$$

quindi abbiano:

$$
\alpha_{0}=\frac{a_{3}}{R_{t b}^{\frac{1}{4}}} \quad \alpha_{1}=-\frac{a_{2}}{R_{. b}^{\frac{1}{4}}} \quad \alpha_{2}=\frac{a_{1}}{R_{c}^{\frac{1}{2}}} \quad \alpha_{3}=-\frac{a_{0}}{R_{c t}^{\frac{1}{2}}} .
$$

Con questi valori la y diviene:

$$
\varphi=\frac{1}{R_{u}^{\frac{1}{3}}}\left|a_{3} d x_{1}^{3}-3 a_{2} d x_{1}^{2} d x_{2}+3 a_{1} d x_{1} d x_{2}^{2}-a_{0} d x_{2}^{3}\right|
$$

Questa cubica ammette $\Delta_{1}[U, V, W]$ come invariante, e mediante di essa, col procedimento del $\S 3$, possiamo determinare le $H_{q r, p}$ in funzione dei coefficienti $\beta$ della sua trasformata, i quali saranno formati coi coefficienti $b$, come i coefficienti di $\varphi$ sono formati colle $a$. I determinanti (14) che devono essere differenti da zero, perchè la determinazione delle $H_{q r}$ sia possibile sono in questo caso:

$$
\frac{1}{R_{\beta}}\left(\beta_{0} \beta_{2}-\beta_{1}^{2}\right) \quad \frac{1}{R_{1}}\left(\beta_{1} \beta_{3}-\beta_{2}^{2}\right) .
$$

La determinazione delle $H_{q r, p}$ si può fare anche mediante una forma quadratica; difatti la $\varphi$ ammette la forma covariante assoluta quadratica:

$$
\psi=\frac{1}{R_{b}^{\frac{1}{3}}}\left\{\left(a_{1} a_{3}-a_{2}^{2}\right) d x_{1}^{2}-\left(a_{0} a_{3}-a_{1} a_{2}\right) d x_{1} d x_{2}+\left(a_{0} a_{2}-a_{1}^{2}\right) d x_{2}^{2}\right\}
$$


la quale ammetterà pure per invariante $\Delta_{1}[U, V, W]$. Possiamo quindi applicare a questa forma il procedimento del paragrafo precedente per determinare le $H_{q r}$. La condizione per la risolubilità delle equazioni corrisponlenti è, in questo caso, che sia differente da zero il discriminante $R_{\alpha}$; esso inoltre dovrà essere supposto positivo, per non introdurre immaginari. Se $R_{\alpha}$ ̀̀ positivo, anche $R_{\beta}$ sarà positivo, qualunque siano le variabili $y$, e da ciò segue pure che $\mathrm{i}$ due determinanti $(\xi)$ non potranno mai essere zero.

Due forme binarie di grado dispari $2 \mu+1$ di coefficienti $v$ e $\%$, ammettono l'invarjante simultaneo rappresentato simbolicamente da:

$$
(\alpha \gamma)^{2 \mu+1}
$$

il quale è lincare nei coefficienti di ciascuna forma; inoltre una forma binaria di grado dispari $\alpha_{i}^{p+1}=\beta_{i}^{q^{\mu+1}}=\cdots$ ammette il covariante quadratico:

$$
(\alpha . \beta)^{2 \mu} \alpha_{x} \beta_{x} \text {. }
$$

Da ciò segue che le considerazioni precedenti sono applicabili a tutte le equazioni di ordine dispari con due varjabili indipendenti, cioè si ha il teorema:

La trasformazione di un'equazione a derivate parziali, lineare, omogenea, a coefficienti costanti, d'ordine dispari $2 \mu+1$, con due variabili indipendenti $\dot{e}$ riducibile in generale a quella di una forma differenziale binaria di grado $2 \mu+1$; inoltre la determinazione delle funzioni $H_{q r, p}$ si può fare mediante $i$ coefficienti di una. forma differenziale binaria quadratica.

Scriviamo infine la forma generale della trasformata dell'equazione del $3{ }^{\circ}$ ordine quale risulta dalle relazioni $(\delta)(\varepsilon)$, oppure dal principio di $\mathrm{J}_{\mathrm{ACOBI}}$.

Cominciamo ad osservare che il gruppo dei covarianti della equazione è jn questo caso costituito dai seguenti due:

$$
\begin{aligned}
\Delta_{1}[U, V, W] & =\sum_{p_{1} r} b_{p q r} \frac{\partial U}{\partial y_{p}} \frac{\partial V}{\partial y_{q}} \frac{\partial W}{\partial y_{r}} \\
\Delta_{2}[U \mid V] & =\sum_{p q r} b_{p q r} \frac{\partial^{2} U}{\partial y_{p} \partial y_{q}} \frac{\partial V}{\partial y_{r}}+\sum_{p q} b_{p q} \frac{\partial U}{\partial y_{p}} \frac{\partial V}{\partial y_{q}} .
\end{aligned}
$$

Se ora si pone:

si ha:

$$
U_{r}=\frac{\partial \Delta_{2}[U|V|}{\partial\left(\frac{\partial V}{\partial y_{r}}\right)}=\sum_{p q} b_{p q r} \frac{\partial^{2} U}{\partial y_{p} \partial y_{q}}+\sum_{p} b_{p r} \frac{\partial U}{\partial y_{p}},
$$

$$
\Delta_{3}[U]=\frac{1}{B} \underset{r}{\mathbf{y}} \frac{\partial}{\partial y_{r}}\left(B U_{r}\right)
$$


Ponendo invece:

si ha:

$$
\begin{aligned}
& U_{p q}^{*}=\frac{\partial^{2} \Delta_{1}[U, V, W]}{\partial\left(\frac{\partial V}{\partial y_{p}}\right) \partial\left(\frac{\partial W}{\partial y_{q}}\right)}=\frac{\partial \Delta_{q}[V \mid U]}{\partial\left(\frac{\partial^{2} V}{\partial y_{p} \partial y_{q}}\right)}=\frac{\mathbf{y}}{\mathbf{r}} b_{p q r} \frac{\partial U}{\partial y_{r}} \\
& U_{p}^{*}=\frac{\partial \Delta_{2}[V \mid U]}{\partial\left(\frac{\partial V}{\partial y_{p}}\right)}=\mathbf{\mathbf { s }} b_{p q} \frac{\partial U}{\partial y_{q}}
\end{aligned}
$$

Infine si ha:

$$
\Delta_{3}[U]=\frac{1}{B} \sum_{p q}^{\mathbf{z}} \frac{\partial^{2}}{\partial y_{p} \partial y_{q}}\left(B U_{p q}^{*}\right)-\frac{1}{B} \sum_{p}^{\mathbf{y}} \frac{\partial}{\partial y_{p}}\left(B U_{p}^{*}\right)
$$

in cui:

$$
\Delta_{2}[U, V]=\frac{1}{B} \sum_{r} \frac{\partial}{\partial y_{r}}\left(B \frac{\partial \Delta_{1}[U, V, W]}{\partial\left(\frac{\partial W}{\partial y_{r}}\right)}\right)
$$

$$
\frac{\partial \Delta_{1}[U, V, W \mid}{\partial\left(\frac{\partial W}{\partial y_{i}}\right)}=\sum_{\perp q} b_{p q}, \frac{\partial U}{\partial y_{p}} \frac{\partial V}{\partial y_{q}} .
$$

\section{$\S 6$. Forme algebriche reciproche.}

Per le espressioni $\Delta_{1}\left[U_{1}, U_{2}, \ldots U_{.}\right]$, quando $\nu$ è pari, è possibile stabilire un procedimento generale per costruire delle forme differenziali di grado $\nu$, rispetto alle quali esse sono invariabili. Prima però è necessario estendere alle forme di ordine pari qualsiasi il concetto ordinario della reciprocità delle forme quadratiche.

Cominciamo a richiamare la definizione delle forme quadratiche reciproche, servendoci della notazione simbolica. Consideriamo il determinante:

e poniamo:

$$
\left(a^{(1)} a^{(2)} \ldots a^{(n)}\right)=\left|\begin{array}{ccc}
a_{1}^{(1)} & a_{1}^{(2)} \ldots & a_{1}^{(n)} \\
a_{2}^{(1)} & a_{2}^{(2)} \ldots & a_{2}^{(n)} \\
\ldots & \ldots & \ldots \\
a_{n}^{(1)} & a_{12}^{(2)} \ldots & a_{n}^{(n)}
\end{array}\right|
$$

$$
\frac{\partial\left(a^{(1)} a^{(2)} \ldots a^{(n)}\right)}{\partial a_{i}^{(1)}}=\left(a^{(z)} a^{(3)} \ldots a^{(n)}\right)_{i}, \ldots \quad \frac{\partial\left(a^{(1)} a^{(2)} \ldots a^{(n)}\right)}{\partial a_{i}^{(n)}}=\left(a^{(2)} a^{(3)} \ldots a^{(n)}\right)_{i} .
$$


Costruiamo ora il determinante:

$$
\left(\alpha^{(1)} \alpha^{(2)} \ldots \alpha^{(n)}\right)=\left|\begin{array}{ccc}
\alpha_{1}^{(1)} & \alpha_{1}^{(2)} \ldots & \alpha_{1}^{(n)} \\
\alpha_{2}^{(1)} & \alpha_{2}^{(2)} \ldots & \alpha_{2}^{(n)} \\
\ldots & \ldots & \ldots \\
\alpha_{1}^{(1)} & \alpha_{i j}^{(2)} \ldots & \alpha_{\mu}^{(n)}
\end{array}\right|,
$$

i cui elementi siano definiti in funzione di quelli del precedente mediante le formule:

$$
\left.\begin{array}{c}
\left(a^{(1)} a^{(2)} \ldots a^{(n)}\right) \alpha_{i}^{(1)}=\left(a^{(2)} a^{(3)} \ldots a^{(n)}\right)_{i} \\
\ldots \ldots \ldots \ldots \ldots \ldots \ldots \ldots \\
\left(a^{(1)} a^{(2)} \ldots a^{(n)}\right) \alpha_{i}^{(1)}=\left(a^{(1)} a^{(2)} \ldots a^{(n-1)}\right)_{i} .
\end{array}\right\}
$$

Avremo allora, come è noto dalla teoria dei determinanti,

$$
\left.\begin{array}{c}
\left(\alpha^{(1)} \alpha^{(2)} \ldots \alpha^{(n)}\right) \alpha_{i}^{(1)}=\left(\alpha^{(2)} \alpha^{(3)} \ldots \alpha^{(n)}\right)_{i} \\
\ldots \ldots \ldots \ldots \ldots \ldots \ldots \\
\left(\alpha^{(1)} \alpha^{(2)} \ldots \alpha^{(n)}\right) \alpha_{i}^{(n)}=\left(\alpha^{(1)} \alpha^{(2)} \ldots \alpha^{(n-1)}\right)_{i} .
\end{array}\right\}
$$

Si abbia ora una forma quadratica ad $n$ variabili:

$$
\varphi:=\sum_{i h} \alpha_{i h} z_{i} z_{h}
$$

oppure simbolicamente:

$$
\varphi=a_{z}^{(1)^{2}}=a_{z}^{(2)^{2}}=\cdots=a_{z}^{\left(u_{z}^{2},\right.},
$$

il cui discriminante, all' infuori di un fattore numerico che, per semplicità, trascuriamo, può essere rappresentato con

$$
a=\left(a^{(1)} a^{(2)} \ldots a^{(n)}\right)^{2} .
$$

Si chiama forma reciproca di $\varphi$, una forma $\Phi$ i cui coefficienti $\alpha_{i h}$ sono definiti simbolicamente da:

$$
\alpha_{i h}=\alpha_{i}^{(r)} \alpha_{h}^{(r)},
$$

ove per $\alpha_{i}^{(r)}, \alpha_{l}^{(\prime \prime)}$ si intendono le espressioni definite dalle (18), ed $r$ è un indice determinato qualunque; è chiaro che il secondo membro della formula precedente ha un significato reale, e non identicamente zero, in funzione dei coefficienti di p. Noi porremo:

oppure simbolicamente:

$$
\Phi=\sum_{i h} \alpha_{i h} Z_{i} Z_{h}
$$

$$
\Phi=\alpha_{\%}^{(1)^{2}}=\alpha_{\%}^{(2)^{2}}=\cdots=\alpha_{z}^{(2,2} .
$$


La ragione della denominazione introdotta per $\Phi$ sta nel fatto che, se noi da $\Phi$ deduciamo una nuova forma quadratica collo stesso procedimento che ha servito a dedurre $\Phi$ da $\varphi$, ritroviamo la $\varphi$. Difatti siano $\alpha_{i h}^{\prime}$ i coefficienti di questa nuova forma; dalla (19) abbiamo subito:

Avremo inoltre:

$$
\alpha_{i h}^{\prime}=a_{i}^{(i)} a_{h}^{(r)}=a_{i h} \text {. }
$$

e reciprocamente:

$$
\left(a^{(1)} a^{(2)} \ldots a^{(n)}\right)^{2} \alpha_{i n}=\frac{1}{2 !} \frac{\partial^{2}\left(a^{\prime} a^{\prime} a^{\prime} \ldots a^{(n)}\right)^{2}}{\partial a_{i}^{(n)} \partial a_{h}^{(n)}},
$$

$$
\left(\alpha^{(1)} \alpha^{(2)} \ldots \alpha^{(n)}\right)^{2} \alpha_{i h}=\frac{1}{2 !} \frac{\partial^{2}\left(\alpha^{(1)} \alpha^{(2)} \ldots \alpha^{(n)}\right)^{2}}{\partial \alpha_{i}^{(n)} \partial \alpha_{h}^{n}} .
$$

Inoltre, essendo uguale all' unità il prodotto dei determinanti (16) (17) arremo anche:

$$
\left(a^{(i)} a^{(2)} \ldots a^{(2)}\right)^{2}\left(\alpha^{(1)} \alpha^{(2)} \ldots \alpha^{(1)}\right)^{2}=1 .
$$

Queste considerazioni possono essere immediatamente estese a qualsiasi forma di grado pari. Sin:

$$
\psi=\sum_{i_{1} i_{2} \ldots i_{2},} a_{i_{1}} i_{2} \ldots i_{2,}, z_{i_{1}} z_{i_{2}} z_{i_{2,}}
$$

onpure simbolicamente:

$$
\varphi=a_{z}^{\left(11^{2 \nu}\right.}=a_{z}^{\left(2,2^{2 \nu}\right.}=\cdots=a_{z}^{(n, 2 \nu}
$$

la forma data di grado $2 \nu$, essa ammette l'invariante di grado $n$ :

$$
a=\left(a^{(1)} a^{(2)} \ldots a^{(n)}\right)^{2 \nu} \text {. }
$$

Definiremo forma reciproca una forma $\Phi$ di grado $2 \nu$, i cui coefficienti $\alpha_{i_{1}} i_{2}, i_{2}$, sono determinati dalla formula:

$$
\alpha_{i_{1}} i_{2} \ldots i_{i_{y}}=\alpha_{i_{1}}^{(j)} \alpha_{i_{9}}^{(i)} \ldots \alpha_{i_{2},}^{(j)},
$$

ove le $\alpha_{i}^{(n)}$ sono le espressioni definite dalle (18), ed $r$ è un indice determinato qualunque; il secondo membro della formula precedente ha un significato reale, e non identicamente zero, in funzione dei coefficienti di $\varphi$. Noi porremo:

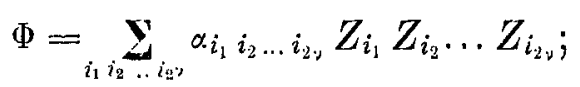

oppure simbolicamentc:

$$
\Phi=\alpha_{z}^{\left(1,{ }^{2 \nu}\right.}=\alpha_{z}^{(2,2 \nu}=\cdots=\alpha_{z}^{(2,2)} .
$$

Se deduciamo da $\Phi$ una nuova forma di grado $2 \nu$, collo stesso procedimento, col quale abbiamo dedotto $\Phi$ da $\varphi$, ritroviamo la $\varphi$. Difatti, indicando 
con $\alpha_{i_{1}}^{\prime} i_{2} \ldots i_{2 \nu}$ i coefficienti di questa nuova forma, dalla (19) abbiamo:

Abbiamo poi:

$$
\alpha_{i_{1} i_{2} \ldots i_{2 \nu}}^{\prime}=a_{i_{1}}^{(\prime)} a_{i_{\mathrm{g}}}^{(\prime)} \ldots a_{i_{2 \nu}}^{(\prime)}=a_{i_{1} i_{2} \ldots i_{2 \nu}}
$$

e reciprocamente:

$$
\left(a^{(1)} a^{(2)} \ldots a^{(n)}\right)^{2 \nu} \alpha_{i_{1} i_{2} \ldots i_{2},}=\frac{1}{2 \nu !} \frac{\partial^{2 \nu}\left(a^{(1)} a^{(2)} \ldots a^{(2 l)}\right)^{2 \nu}}{\partial a_{i_{1}}^{(r)} \partial a_{i_{1}}^{(r)} \ldots \partial a_{i_{2} \nu}^{(r)}}
$$

$$
\left(\alpha^{(1)} \alpha^{(2)} \ldots \alpha^{(n)}\right)^{2 \nu} a_{i_{1} i_{2} \ldots i_{2},}=\frac{1}{2 \nu !} \frac{\partial^{2 \nu}\left(\alpha^{(1)} \alpha^{2)} \ldots \alpha^{(n i}\right)^{2 \nu}}{\partial \alpha_{i_{1}}^{(p)} \partial \alpha_{i_{2}}^{(p)} \ldots \partial \alpha_{i_{2} \nu}^{(r)}} .
$$

Finalmente, se formiamo per la $\Phi$ l'invariante $\alpha$ analogo all' invariante $a$ della $\varphi$,

$$
\alpha=\left(\alpha^{(1)} \alpha^{(2)} \ldots \alpha^{(n)}\right)^{2 \nu} \text {, }
$$

osservando che il prodotto dei due determinanti (16) (17) è uguale all' unità, avremo:

$$
a \alpha=1 \text {. }
$$

In tutte queste considerazioni naturalmente si deve supporre che la forma $\varphi$ abbia l'invariante a differente da zero.

Ricordando la formula di Eudero per la espressione di una funzione omogenea di $n$ variabili mediante le derivate di ordine $2 \nu$, e osservando la (20) si trova:

$$
\Phi=\frac{\left(a^{(1)} \ldots a^{\prime p-1} Z a^{(v+1)} \ldots a^{\prime n}\right)^{2 y}}{\left(a^{1 !} a^{(2 !} \ldots a^{(n)}\right)^{2 y}},
$$

e cosi per la (21) si ha anche:

$$
\varphi=\frac{\left(\alpha^{1} 1 \ldots \alpha^{\prime} r-1\right.}{\left.\alpha^{1} z \alpha^{(r+1)} \ldots \alpha^{(n i}\right)^{2)}} .
$$

La prima di queste formule mostra che la $\Phi$ è una forma contravariante di $\varphi$ in modo assoluto.

Per avere un'idea delle legge di formazione dei coefficienti della forma reciproca possiamo osservare per es. che i coefficienti della reciproca di una ternaria quadratica, se si fa astrazione del denominatore comune, sono determinanti di $2 .^{\circ}$ ordine, cioè hanno forma analoga a quella dell' invariante $(a b)^{2}$ di una binaria quadratica; i coefficienti della reciproca di una quartica ternaria hanno forma analoga a quella dell' invariante $(a b)^{4}$ di una quartica binaria, ecc.; in generale i coefficienti della reciproca di una $n$-aria di grado $2 \nu$ sono formati in modo analogo all' invariante $\left(a^{(1)} a^{(2)} \ldots a^{(n-1)}\right)^{2 v}$ di una $(n-1)$-aria, sempre all' infuori del denominatore comune $\left(a^{(1)} a^{(2)} \ldots a^{(n)}\right)^{2 *}$.

Annali di Matematica, tomo XVIIT. 
Dalle considerazioni precedenti e dall'osservazione che le derivate prime di una funzione di $n$ variabili sono controgredienti ai differenziali delle variabili stesse, risulta subito che data un' espressione:

$$
\Delta_{1}\left[U_{1}, U_{2}, \ldots U_{2 v}\right]==\sum_{i_{1} i_{2} \ldots i_{2},}^{y} a_{i_{1} i_{2}}, i_{2 y} \frac{\partial U_{1}}{\partial x_{i_{1}}} \ldots \frac{\partial U_{2 v}}{\partial x_{i_{2 v}}} .
$$

si otterrà una forma differenziale di grado 2 v rispetto alla quale tale espressione sarà invariabile, costruendo la forma differenziale reciproca della precedente, considerata come forma $2 \nu$-plo lineare delle derivate delle funzioni $U_{1}, \ldots U_{2 v}$.

Inversamente, data una forma differenziale di grado $2 \%$, lo stesso procedimento condurrà ad una espressione $\Delta_{1}\left[U_{1}, \ldots U_{2 y}\right]$ invariabile rispetto alla forma.

\section{$\S 7$. Equazioni del $4 .^{\circ}$ ordine.}

Le formule pel cambiamento delle variabili indipendenti per la trasformazione della espressione:

ci dànno:

$$
\Delta_{4}[U]=\sum_{i \hbar k l} a_{i h k l} \frac{\hat{c}^{4} U}{\partial x_{i} \partial x_{h} \partial x_{k} \partial x_{l}},
$$

$$
\begin{aligned}
& \Delta_{4}[U]=\sum_{p q_{s}} \Delta_{1}\left[y_{p}, y_{q}, y_{r}, y_{s}\right] \frac{\partial^{4} U}{\hat{\imath} y_{p} \partial y_{q} \partial y_{1} \partial y_{s}}+ \\
& +6 \sum_{p_{q} r}^{\mathbf{y}_{2}} \Delta_{2}\left[y_{p} \mid y_{q}, y_{r}\right] \frac{\hat{o}^{3} U}{\partial y_{p} \partial y_{q^{2}} \dot{r}^{2}} \\
& +\sum_{p q u}^{\mathbf{s}}\left(3 \Delta_{2}\left[y_{p}, y_{q}\right]+4 \Delta_{3}\left[y_{p} \mid y_{q}\right]\right) \frac{\check{C}^{2} U}{\partial y_{p} \partial y_{q}}+\sum_{p} \Delta_{4}\left[y_{p}\right] \frac{\partial U}{\partial y_{p}},
\end{aligned}
$$

dove abbiamo:

$$
\begin{aligned}
& \Delta_{1}\left[y_{p}, y_{q}, y_{r}, y_{s}\right]=\sum_{i k l}^{\mathbf{z}} a_{i h k l} \frac{\partial y_{p}}{\partial x_{i}} \frac{\partial y_{q}}{\partial x_{l}} \frac{\partial y_{r}}{\partial x_{k}} \frac{\partial y_{s}}{\partial x_{l}}=b_{p q r_{s}}
\end{aligned}
$$

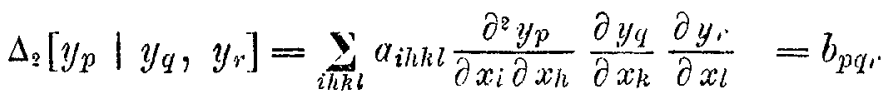

$$
\begin{aligned}
& \Delta_{2}\left[y_{p}, y_{q}\right]=\underset{i \hbar k_{l} l}{a_{i h k l}} \frac{\partial^{2} y_{p}}{\partial x_{i} \partial x_{h}} \frac{\partial^{2} y_{q}}{\partial x_{k} \partial x_{l}}=b_{p q} \\
& \Delta_{3}\left[y_{p} \mid y_{q}\right]=\sum_{i \hbar k l} a_{i h k l} \frac{\partial^{3} y_{p}}{\partial x_{i} \partial x_{h} \partial x_{l}} \frac{\partial y_{q}}{\partial x_{l}}=b_{p q}^{\prime} \\
& \Delta_{4}\left[y_{p}\right]=\sum_{i k k l} a_{i h k l} \frac{\partial^{4} y_{p}}{\partial x_{i} \partial x_{h} \partial x_{k} \partial x_{l}}=b_{p} .
\end{aligned}
$$


Col metodo che abbiamo indicato per stabilire la $\left(10^{\prime}\right)$ si trova:

$$
\begin{aligned}
& b_{p q r}=\sum_{l m} H_{l m, p} b_{l m q r} \\
& b_{p q}=\sum_{l m n i} H_{l m, p} H_{n i, q} b_{l m n i} \\
& b_{p q}^{\prime}=\sum_{l m n} H_{l m n p} b_{l m n q} \\
& b_{p}=\sum_{l m u n i} H_{l m n i p} b_{l m n i}
\end{aligned}
$$

Dalle (5) poi abbiamo:

$$
\left.\begin{array}{l}
H_{l m n, p}=\frac{\partial H_{l m, p}}{\partial y_{n}}+\sum_{k}\left(I_{l n, k} H_{m k, p}+H_{n n, k} H_{l k p}\right) \\
H_{l m n i_{,} p}=\frac{\partial H_{l m n, p}}{\partial y_{i}}+\sum_{i}\left(H_{l i, k} H_{m n k} p+H_{m i p} H_{n l k p}+H_{n i, k} H_{l n k, p}\right),
\end{array}\right\}
$$

c finalmente dalla (11):

$$
\frac{\partial b_{p q u s}}{\partial y_{t}}=\sum_{t}\left(H_{l t p} b_{l q r s}+H_{l t, q} b_{l r s p}+H_{l t}, b_{l s p q}+H_{l t, s} b_{l p q r}\right) .
$$

Il problema, secondo il metodo generale, si riduce alla eliminazione delle $H_{p q, r}$ dalle $(\alpha)(\beta)$ mediante le $(y)$.

I covarianti del gruppo dell' equazione in questo caso sono quattro; le loro espressioni colle variabili $y$ sono:

$$
\begin{aligned}
& \Delta_{1}[U, V, W, T]=\underset{p q r^{\prime} s}{\mathbf{\Sigma}} \Delta_{1}\left[y_{p}, y_{q}, y_{r}, y_{s}\right] \frac{\partial U}{\partial y_{p}} \frac{\partial V}{\partial y_{q}} \frac{\partial W}{\partial y_{r}} \frac{\partial T}{\partial y_{s}} \\
& \Delta_{i}[U \mid V, W]=\sum_{p \eta^{\prime} s}^{\mathbf{S}_{1}} \Delta_{1}\left[y_{p}, y_{q}, y_{r}, y_{s}\right] \frac{\partial^{z} U}{\partial y_{p} \partial y_{q}} \frac{\partial V}{\partial y_{r}} \frac{\partial W}{\partial y_{s}}+ \\
& +\sum_{p q r} \Delta_{2}\left[y_{p} \mid y_{q}, y_{r}\right] \frac{\partial U}{\partial y_{p}} \frac{\partial V}{\partial y_{q}} \frac{\partial W}{\partial y_{r}} \\
& \Delta_{2}[U, V]=\sum_{p, r_{s}}^{\Delta_{1}} \Delta_{1}\left[y_{p}, y_{q}, y_{r}, y_{s}\right] \frac{\hat{\partial}^{2} U}{\partial y_{p} \partial y_{q}} \frac{\hat{\partial}^{2} V}{\partial y_{r} \partial y_{s}}+ \\
& +\sum_{p q r} \Delta_{z}\left[y_{p} \mid y_{q}, y_{r}\right]\left(\frac{\partial^{2} U}{\partial y_{q} \partial y_{r}} \frac{\partial V}{\partial y_{p}}+\frac{\partial^{2} V}{\partial y_{q} \partial y_{r}} \frac{\partial U}{\partial y_{p}}\right)+ \\
& +\sum_{p q} \Delta_{2}\left[y_{p}, y_{q}\right] \frac{\partial U}{\partial y_{p}} \frac{\partial V}{\partial y_{q}} \\
& \Delta_{3}[U \mid V]=\underset{p q r s}{\sum} \Delta_{1}\left[y_{p}, y_{q}, y_{r}, y_{s}\right] \frac{\partial^{3} U}{\partial y_{p} \partial y_{q} \partial y_{r}}+ \\
& +3 \underset{p q^{r}}{\sum_{2}} \Delta_{2}\left[y_{p} \mid y_{q}, y_{r}\right] \frac{\partial^{2} U}{\partial y_{p} \partial y_{q}} \frac{\partial V}{\partial y_{r}}+\sum_{p q} \Delta_{3}\left[y_{p} \mid y_{q}\right] \frac{\partial U}{\partial y_{p}} \frac{\partial V}{\partial y_{q}} .
\end{aligned}
$$


Si può osservare che tutte queste espressioni, al pari di quella della $\Delta_{4}[U]$, in virtù delle $\left(10^{\prime}\right)$ si possono rappresentare come funzioni lineari delle $b_{\text {pqrs }}$.

Le relazioni che si deducono dalle (a) derivando rispetto alle $y$, e sostituendo poi alle derivate delle $H_{l m, p}, H_{l m n, p}$ e delle $b_{l m n i}$ i valori dati dalle $(\beta)(\gamma)$ sono le seguenti:

ove possiamo prendere:

$$
\begin{aligned}
b_{q, s}+b_{r s q}+b_{s q r} & =\frac{1}{B} \frac{\mathbf{y}}{p} \frac{\partial}{\partial y_{p}}\left(B b_{p q r s}\right) \\
b_{p q}^{\prime}+b_{p q} & =\frac{1}{B} \mathbf{y} \frac{\partial}{\partial y_{r}}\left(B b_{p q r}\right) \\
b_{p} & =\frac{1}{B} \sum_{q} \frac{\partial}{\partial y_{q}}\left(B b_{p q}^{\prime}\right)
\end{aligned}
$$

$$
B=\frac{1}{4} \frac{\bar{b}}{b}
$$

indicando con $b$ l'invariante rappresentato simbolicamente da $\left(b^{(1)} b^{(2)} \ldots b^{(n)}\right)^{4}$.

Per quanto abbiamo visto nel paragrafo precedente otterremo poi una forma differenziale di $4 .^{\circ}$ ordine, rispetto alla quale la forma $\Delta_{1}[U, V, W, T]$ sarà invariabile, costruendone la forma differenziale a coefficienti reciproci. Nel seguente paragrafo vedremo meglio le relazioni fra questa forma ed $i$ covarianti dell'equazione; qui mi limiterò a dare la forma della trasformata dell'equazione, quale risulta dal principio di $\mathrm{J}_{\mathrm{ACOBI}}$ oppure dalle relazioni $(0)$. Come nel caso dell'equazione di $3 .^{\circ}$ ordine abbiamo trorato due forme della trasformata, qui invece ne abbiamo tre.

Se si pone:

$$
U_{s}=\frac{\partial \Delta_{3}[U \mid V]}{\partial\left(\frac{\partial V}{\partial y_{*}}\right)}=\sum_{p q r} b_{x q r s} \frac{\partial^{3} U}{\partial \eta_{p} \partial y_{q} \partial y_{r}}+3 \sum_{p_{q}} b_{p q s} \frac{\partial^{2} U}{\partial y_{p} \partial y_{q}}+\sum_{p} b_{p s}^{\prime} \frac{\partial U}{\partial y_{p}}
$$

si ha:

$$
\Delta_{4}[U]=\frac{1}{B} \frac{y}{8}\left(B U_{s}\right)
$$

Ponendo invece:

$$
\begin{aligned}
& U_{p q}^{*}=\frac{\partial \Delta_{2}}{\partial\left(\frac{U, V]}{\partial y^{2} V}\right)}=\sum_{r s} b_{p q r s} \frac{\partial^{2} U}{\partial y_{p} \partial y_{q} \partial y_{s}}+\sum_{r} b_{r p q} \frac{\partial U}{\partial y_{i}} \\
& U_{p}^{*}=\frac{\partial \Delta_{2}[U, V]}{\partial\left(\frac{\partial V}{\partial y_{p}}\right)}=\sum_{r s}^{\mathbf{y}} b_{p r s} \frac{\partial^{2} U}{\partial y_{r} \partial y_{s}}+\sum_{r}^{\mathbf{y}} b_{r p} \frac{\partial U}{\partial y_{r}}
\end{aligned}
$$


si trova:

Finalmente ponendo:

$$
\Delta_{i}[U]=\frac{1}{B} \sum_{2, q} \frac{\partial^{2}}{\partial y_{p} \partial y_{q}}\left(B U_{p q}^{*}\right)-\frac{1}{B} \underset{p}{y_{1}}\left(B U_{p}^{*}\right)
$$

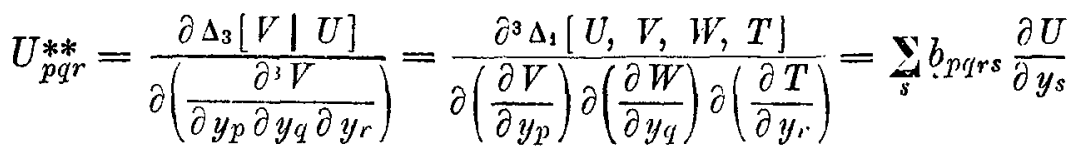

$$
\begin{aligned}
& U_{p q}^{* *}=\frac{\partial \Delta_{3}|V| U \mid}{\partial\left(\frac{\partial V}{\partial y_{p} \partial y_{p}}\right)}=\sum_{s} b_{p q s} \frac{\partial U}{\partial y_{s}} \\
& U_{p}^{* *}==\frac{\partial \Delta_{3}[V \mid U]}{\partial\left(\frac{\partial V}{\partial y_{p}}\right)}=\sum_{s} b_{p^{\prime}}^{\prime} \frac{\partial U}{\partial y_{s}}
\end{aligned}
$$

si ha:

$$
\begin{aligned}
& \Delta_{4}[U]=\frac{1}{B} \sum_{p q r}^{\Sigma} \frac{\hat{c}^{3}}{\partial y_{p} \partial \bar{y}_{q} \partial \bar{y}_{i}}\left(B U_{p q r}^{* *}\right)-\frac{1}{B} \sum_{p_{q}} \frac{\hat{\partial}^{2}}{\partial ! / p \partial y_{q}}\left(B U_{p q}^{* *}\right)+ \\
& +\frac{1}{B} \frac{\mathrm{y}}{p} \frac{\partial}{\partial y_{p}}\left(B U_{p}^{* *}\right)
\end{aligned}
$$

Altre relazioni fra i quattro covarianti si possono ottenere assai facilmente col principio di $\mathrm{J}_{\text {ACOBI. }}$

\section{§ 8. Parametri differenziali.}

Quelle espressioni, che nei paragrafi precedenti abbiamo chiamato covarianti dell'equazione:

$$
\Delta \cdot[U]=0
$$

allorchè esiste una forma differenziale rispetto alla quale le espressioni del $1 .^{\circ}$ ordine $\Delta_{1}\left[U_{1}, \ldots, U_{v}\right]$ sono invariabili, godono tutte di proprietà invariantiva rispetto alla forma stessa, e possono quindi essere chiamati parametri differenziali di questa, per analogia colla denominazione in uso per le forme quadratiche. Le forme, che abbiamo considerato pero non erano generali, ma implicitamente era ammessa la condizione che fossero riducibili, con opportune trasformazioni di variabili, ad essere a coefficienti costanti. Ora possiamo affrancarci anche da questa restrizione e dare un procedimento generale per la formazione dei parametri di qualunque forma differenziale. Tale procedi- 
mento non è che la estensione, mediante una osservazione assai semplice, del metodo trovato dal prof. Ricor pel caso delle forme quadratiche, e può essere soggetto soltanto a quella restrizione a cui abbiamo accennato nel $\S 3$.

Consideriamo una forma differenziale:

$$
\varphi=\sum_{i_{1} i_{2} \ldots i_{\nu}}^{\mathbf{y}} a_{i_{1} i_{2} \ldots i_{\nu}} d x_{i_{1}} \ldots d x_{i_{\nu}}
$$

di grado 2, i cui coefficienti siano funzioni delle variabili $x$; mutando le variabili $x$ in altre variabili $y$, si ottenga:

$$
\varphi=\sum_{i_{1} i_{2} \ldots i_{\nu}} b_{i_{1}} i_{2} \ldots i_{y} d y_{i_{1}} \ldots d y_{i_{y}}
$$

e mutando similmente le $x$ in altre variabili $z$ si abbia:

$$
\varphi=\underset{i_{1} i_{2} \ldots i_{\nu}}{\mathbf{y}} b_{i_{1} i_{2} \ldots i_{\nu}}^{\prime} d z_{i_{1}} \ldots d z_{i_{\nu}} .
$$

Poniamo come noi paragrafi precedenti:

$$
H_{q r, p}=\sum_{i h} \frac{\partial^{2} y_{p}}{\partial x_{i} \partial x_{h}} \frac{\partial x_{i}}{\partial y_{q}} \frac{\partial x_{h}}{\partial y_{r}},
$$

e inoltre:

$$
H_{q \cdot, p}^{\prime}=\sum_{i h} \frac{\partial^{2} z_{p}}{\partial x_{i} \partial x_{h}} \frac{\partial x_{i}}{\partial z_{q}} \frac{\partial x_{h}}{\partial z_{r}}
$$

Sappiamo che queste espressioni $H_{q r, p}, H_{q r, p}^{\prime}$ si potranno in gencrale rappresentare in modo identico mediante le (15) (15), come funzioni dei coefficienti $b$ e delle loro derivate, e come funzioni dei coefficienti $b^{\prime}$ e delle loro derivate, rispettivamente. Cerchiamo ora quali relazioni esistono fra le $H_{q r} p$ e le $H_{q r, p}^{\prime}$.

La (23') può essere scritta nel seguente modo:

$$
H_{q^{\prime}, p}^{\prime}=\sum_{i h}\left(\sum_{l m} \frac{\partial^{2} z_{p}}{\partial y_{l} \partial y_{m}} \frac{\partial y_{l}}{\partial x_{i}} \frac{\partial y_{m}}{\partial x_{h}}+\sum_{h}^{\mathbf{y}} \frac{\partial z_{p}}{\partial y_{k}} \frac{\partial^{2} y_{k}}{\partial x_{i} \partial x_{h}}\right) \frac{\partial x_{i}}{\partial z_{q}} \frac{\partial r_{h}}{\partial z_{r}},
$$

ossia:

$$
H_{q r p}^{\prime}=\sum_{l m}\left(\frac{\partial^{2} z_{p}}{\partial y_{l} \partial y_{: n}}+\sum_{k} H_{l m, k} \frac{\partial z_{p}}{\partial y_{k}}\right) \frac{\partial y_{l}}{\partial z_{q}} \frac{\partial y_{m}}{\partial z_{r}},
$$

da cui si ha:

$$
\underset{q^{r}}{\sum_{q^{\prime}, p}} H^{\prime} \frac{\partial z_{q}}{\partial y_{i}} \frac{\partial z_{r}}{\partial y_{s}}=\frac{\partial^{2} z_{p}}{\partial y_{i} \partial y_{s}}+\sum_{k} H_{i s, k} \frac{\partial z_{p}}{\partial y_{k}} \text {. }
$$

Stabilita questa formula, supponiamo che si conosca una forma differenziale 
di grado $\lambda$ :

$$
\Phi=\underset{i_{1} i_{3} \ldots i_{\lambda}}{\mathbf{y}} P_{i_{1} i_{2} \ldots i_{\lambda}} d x_{i_{1}} \ldots d x_{i_{\lambda}}
$$

la quale sia covariante di $\varphi$, ed $\mathrm{i}$ cui coefficienti siano funzioni delle $a_{i_{1} \ldots i}$, e delle loro derivate, ed inoltre delle derivate di una o più funzioni arbitrarie $U, V, \ldots$ Nelle due trasformate di $\Phi$ nelle variabili $y$ e $z$

$$
\begin{aligned}
& \Phi=\sum_{i_{1} i_{2} \ldots i_{\lambda}}^{\mathbf{\Sigma}} Q_{i_{1} i_{2} \ldots i_{\lambda}} d y_{i_{1}} \ldots d y_{i_{\lambda}} \\
& \Phi=\sum_{l_{1} l_{2} \ldots l_{\lambda}}^{\mathbf{\Sigma}} Q_{l_{1} l_{2} \ldots l_{\lambda}}^{\prime} d z_{l_{1}} \ldots d z_{l_{\lambda}},
\end{aligned}
$$

i coefficienti saranno, per ipotesi, formati in modo identico ai coefficienti $P_{i_{1} . .} i_{\lambda}$ coi coefficienti delle (22) (22') e colle derivate di questi e delle funzioni $U$, $V, \ldots$ rispetto alle variabili $y$ e $z$ rispettivamente. Fra i coefficienti $Q_{i_{1} \ldots i}$ e $Q^{\prime} l_{1}, l_{\lambda}$ poi avremo le relazioni:

$$
Q_{i_{1} i_{z} \ldots i_{\lambda}}=\sum_{l_{1} l_{2} \ldots l_{\lambda}}^{\mathbf{\Sigma}} Q^{\prime} l_{1} l_{2} \ldots l_{\lambda} \frac{\partial z l_{1}}{\partial y i_{i_{1}}} \cdots \frac{\partial z l_{\lambda}}{\partial y i_{\lambda}} .
$$

Derivando $\mathrm{i}$ due membri rispetto ad $y_{i}$ avremo:

$$
\begin{aligned}
& \frac{\partial Q_{i_{1} i_{2} \ldots i_{\lambda}}}{\partial y_{i}}=\sum_{l l_{1} \ldots l_{\lambda}} \frac{\partial Q^{\prime} l_{1} l_{2} \ldots l_{\lambda}}{\partial z_{l}} \frac{\partial z l}{\partial y i} \frac{\partial z l_{2}}{\partial y i_{1}} \cdots \frac{\partial z l_{\lambda}}{\partial y_{i_{\lambda}}} \\
& +\underset{l_{1} l_{2} \ldots l_{\lambda}}{\mathbf{\Sigma}} Q_{l_{1} l_{2} \ldots l_{\lambda}}^{\prime}\left\{\frac{\partial^{2} z_{l_{1}}}{\partial y_{i} \partial y_{i_{1}}} \frac{\partial z z_{2}}{\partial y i_{2}} \cdots \frac{\partial z l_{\lambda}}{\partial y_{i_{2}}}+\cdots+\right. \\
& \left.+\frac{\partial z l_{1}}{\partial y_{i_{1}}} \cdots \frac{\partial z l_{\lambda_{-1}}}{\partial y_{i_{\lambda_{-1}}}} \frac{\partial^{2} z l_{l_{2}}}{\partial y_{i} \partial y_{i_{\lambda}}}\right\}
\end{aligned}
$$

Eliminando ora dal secondo membro le derivate seconde delle $z$ rispetto alle $y$ mediante la (24) e posto:

$$
\begin{aligned}
& Q_{i i_{1} \ldots \lambda_{\lambda}}=\frac{\partial Q_{i_{1} \ldots i_{\lambda}}}{\partial y_{i}}+\sum_{k} \sum_{j=1}^{\lambda} Q_{i_{1} \ldots i_{j}, k i_{j+1} \ldots \lambda} H_{i_{j}, k} \\
& Q^{\prime} l l_{1} \ldots l_{\lambda}=\frac{\partial Q^{\prime} l_{1} \ldots l_{\lambda}}{\partial y l}+\sum_{k} \sum_{s=1}^{\lambda} Q_{l_{1}, l_{s-1} k l_{s+1} \ldots l_{\lambda}} H^{\prime} l_{l_{s}, k},
\end{aligned}
$$

si trova:

$$
Q_{i i_{1} \ldots i_{\lambda}}=\sum_{l_{1} \ldots l_{\lambda}} Q^{\prime} l_{l_{1} \ldots l_{\lambda}} \frac{\partial z l}{\partial y_{i}} \frac{\partial z l_{1}}{\partial y_{i_{1}}} \cdots \frac{\partial z)}{\partial y i_{\lambda}}
$$


Da questa formula risulta il teorema: La forma di grado $\lambda+1$

$$
\Psi=\underset{i i_{1} \ldots i_{\lambda}}{\mathbf{\Sigma}} Q_{i_{i_{1}} \ldots i \lambda} d x_{i} d x_{i_{1}} \ldots d x_{i,}
$$

i cui coefficienti sono dedotti da quelli di $\Phi$ mediante le (25) i, come $\Phi$, covariante di $\varphi$. Di qui si ha un procedimento per dedurre da una data forma covariante una serie di altre forme covarianti, di grado successivamente crescente di una unità.

Nella Memoria: Ueber die Transformation der homogenen Differentialausdriiclie zweiten Grades (Crelle, Bd. 70), Cunistorrsu ha dimostrato un tcorema perfettamente analogo al precedente $\left({ }^{*}\right)$, nel quale però, al posto delle $H_{q r}$ p compaiono i valori che per queste quantità abbiamo trovato al $\S 4$ nel caso delle forme quadratiche; il teorema vale quindi soltanto pel caso in cui ì quadratica. Noi, avendo sostituito le $H_{q r, p}$, abbiamo reso il teorema applicabile a forme di qualsiasi grado.

Se ora $U$ è una funzione arbitraria delle variabili $x_{1}, \ldots x_{n}$, e poniamo:

$$
U_{r}=\frac{\partial U}{\partial x_{r}}
$$

la forma lincare

$$
\Delta U=\sum_{r} U_{r} d x_{r}
$$

che rappresenta il differenziale completo del $1 .^{\circ}$ ordine della funzione $U$, può sempre essere considerata come covariante di $\varphi$; applicando and essa il teorema di Curistofres generalizzato, potremo dedurne altre forme di grado successivamente crescente di una unità, i cui coefficienti conterranno le derivate parziali della $U$ di ordine uguale al grado della forma, ed inoltre le $a_{i_{1}}, i$, e le loro derivate. Noi porremo secondo la (25):

$$
U_{r r_{1} \ldots r_{\lambda}}=\frac{\partial U_{r_{1} \ldots \cdot \lambda}}{\partial x_{r}}+\sum_{k} \sum_{j=1}^{\prime} U_{n_{1} \ldots r_{j-1} k r_{j+1} \ldots r_{\lambda}} H_{r, r_{j}, k},
$$

ove si intende che le $H_{r_{r_{j}} k}$ siano espresse mediante le $a_{i_{1} \ldots i_{v}}$ e le loro de. rivate, come abbiamo visto al $\S 3$; e avremo allora che la m-csima forma covariante di $\varphi$ sarà:

$$
\Delta^{1 i \mathrm{~L}} U=\sum_{r_{1} r_{2} \ldots r_{m}}^{\mathbb{2}} U_{r_{1} r_{2} \ldots r_{m}} d x_{r_{1}} \ldots d x_{r_{m}}
$$

(*) Chrisforfer considera invece della forma $\boldsymbol{\Phi}$ di grado $\lambda$, una forma $\lambda$ rolte lineare rispetto a $\lambda$ serie di differenziali delle variabili; noi, per sernplicita, non abbiamo introdotto questa estensione, che del resto è evidente potersi fare anclie nel nostro caso. 
Tutti gli invarianti algebrici simultanei della forma $\rho$ e delle

$$
\Delta U, \quad \Delta^{2} U, \ldots \quad \Delta^{m} U, \ldots,
$$

saranno altrettante espressioni invariabili rispetto a $\varphi$ per qualsiasi trasformazione di variabili (ossia parametri differenziali di $\varphi$ ) salvo al più quella restrizione, a cui accennato al $\S 3$, relativamente alle condizioni per la risolubilità delle equazioni, da cui si devono ricavare le $H_{r_{j}, k}$.

Nel caso in cui $\varphi$ è di $2 .^{\circ}$ grado il metodo precedente per la formazione dei parametri coincide con quello citato del prof. RIccr.

Insieme alle serie delle forme (26), potremo considerare altre serje analoghe formate con altre funzioni arbitrarie $V, W, \ldots$ ed ottenere cosi dei parametri dipendenti da parecchie funzioni.

Moltiplicando fra loro le forme di $\nu$ di queste serie si potrà formare un numero finito di forme di grado $\nu$, uguale al grado della forma differenziale data; chiameremo gruppo principale dei parametri l'insieme di quelli che si ottengono dalla considerazione degli invarianti simultanei di questo sistema di forme. Una qualunque di queste forme sarà:

e dovrà essere:

$$
\varphi_{p}=\Delta^{\lambda_{1}} U_{1} \Delta^{\lambda_{2}} U_{2} \ldots \Delta^{\lambda_{p}} U_{f},
$$

$$
\lambda_{1}+\lambda_{2}+\cdots+\lambda_{P}=\nu \text {. }
$$

Considerando come identiche alla $\varphi_{p}$, quelle forme che si ottengono da essa permutando fra lorn le funzioni $O_{1}, \ldots U_{\nu}$, il numero delle forme che compongono il sistema sarà uguale a quello delle soluzioni intere della equazione precedente, per $\rho=1,2, \ldots \nu$.

Come applicazione, costruiamo le forme del gruppo principale per $\nu=4$. Sia :

$$
\varphi=\sum_{i \hbar k l} a_{i h k l} d x_{i} d x_{h} d x_{k} d x_{l}
$$

la forma data di $4 .^{\circ}$ grado; si hanno in questo caso 5 forme del gruppo principale:

$$
\begin{aligned}
& \varphi_{1}=\Delta U \Delta V \Delta W \Delta S \quad \varphi_{2}^{\prime}=\Delta^{2} U \Delta^{2} V \quad \varphi_{4}=\Delta^{4} U \\
& \varphi_{2}=\Delta^{2} U \Delta V \Delta W \quad \varphi_{3}=\Delta^{3} U \Delta V . \quad \text {; }
\end{aligned}
$$

La ammette l'invariante rappresentato simbolicamente da

$$
\left(a^{(1)} a^{(2)} \ldots a^{(n)}\right)^{4}=a, .
$$

che supporremo differente da zero. Se nella espressione simbolica di $a$, al Annali di Matematica, tomo XVIII. 
posto di una serie di simboli $a_{1}^{(i)}, \ldots a_{u}^{(i)}$, sostituiamo un' altra serie $C_{1}^{(i)}, \ldots C_{n}^{(i)}$ di simboli relativi ad un'altra forma di $4 .^{\circ}$ grado di coefficienti $C_{i h l}$, e formiamo l'espressione simbolica:

$$
\frac{1}{a}\left(a^{(1)} \ldots a^{(i-1)} C^{(i)} a^{(i+2)} \ldots a^{(n)}\right)^{4},
$$

questa rappresenterc̀ un invariante simultaneo delle due forme, il quale è lineare nei coefficienti $C_{i h k l}$, e sarà quindi della forma:

$$
\sum_{i h k l} A_{i h k l} C_{i h k l}
$$

Le $A_{i h k l}$ saranno i coefficienti della forma reciproca di $\varphi$. Se per forma di coefficienti $C_{i h h l}$ prendiamo successivamente ciascuna delle (27) otteniamo 5 parametri, che sono lineari nei coefficienti di queste forme, e che possiamo rappresentare con:

$$
\Delta_{1}[U, V, W, S], \quad \Delta_{2}[U \mid V, W], \quad \Delta_{2}[U, V], \quad \Delta_{3}[U \mid V], \quad \Delta_{4}[U] .
$$

Supponiamo che le $A_{i l k l}$ coincidono colle $b_{p q r s}$ del $\S 7$, e le variabili $x$ colle $y$ dello stesso paragrafo; allora la $\varphi$ coinciderà colla forma, i cui coefficienti sono reciproci delle $b_{\text {pqrs }} . L^{\prime}$ invariante $\left(28^{\prime}\right)$ diviene:

$$
\sum_{p q q^{2} s} b_{p q r s} C_{p q r s}
$$

ed i cinque parametri ora trovati si possono scrivere:

$$
\begin{aligned}
& \Delta_{1}[U, V, W, T]=\underset{p q r^{\prime} s}{\Sigma} b_{p q_{r s}} U_{p} V_{q} W_{r} T_{s} \\
& \Delta_{2}[U, V, W]=\sum_{p q^{\prime} s}^{\mathbf{y}} b_{p q \cdot s} U_{p q} V_{r} W_{s} \\
& \Delta_{i}[U, V]=\sum_{p q q s} b_{p q r s} U_{p q} V_{r s s} \\
& \Delta_{3}[U \mid V]=\sum_{1, q, s} b_{p q r s} U_{p q u} \cdot V_{s} \\
& \Delta_{1}[U]=\sum_{p q^{r s}} b_{p q r s} U_{p q r s}
\end{aligned}
$$

ove le $U_{p}, U_{p q}, \ldots V_{r}, \ldots$ sono determinati colla legge che risulta dalla (25"). $\grave{\mathbf{E}}$ ora assai facile verificare che le espressioni dei secondi membri coincidono con quelle rappresentate colle notazioni dei primi membri nel $\S 4$, cioè colle trasformate del primo membro dell'equazione del $4 .^{\circ}$ ordine e dei suoi quattro covarianti. La invariabilità di queste espressioni resta cos̀̀ dimostrata, salvo le solite condizioni, senza la limitazione della riducibilità a coefficienti costanti. 
Questi risultati completano quelli a cui jo era arrivato considerando le forme differenziali binarie del $4 .^{\circ}$ grado, rispetto alle trasformazioni lineari (tomo 18 di questi Arnali).

Se le variabili sono in numero maggiore di 2 , le due forme di coefficienti $a_{i h k l}, C_{i h k l}$, oltre l'invariante simultaneo (28), hanno tutti quelli che si ottengono dalla espressione simbolica:

$$
\frac{1}{a}\left(a^{(1)} \ldots a^{(i)} C^{(i+1)} \ldots C^{(i)}\right)^{4},
$$

facendo $i=0,1,2, \ldots, n-2$. Supponendo che $\mathrm{i}$ simboli $C$ si riferiscano ai coefficenti delle forme (27), otteniamo oltre i parametri (29) che sono lineari nei coefficienti di queste forme, altri $5(n-1)$ parametri, che contengono i coefficienti delle forme stesse ai gradi $n, n-1, \ldots 3,2$.

$\grave{E}$ chiaro che risultati analoghi si possono trovare per tutte le forme di grado pari.

$$
\text { Luglio, } 1890 .
$$

Correzione. Le formule generali del $\S 8$ stanno, come è detto in principio del paragrafo stesso, per forme differenziali, i cui coefficienti sono funzioni qualsiansi delle variabili indipendenti; ma non è esatto asserire che il procedimento indicato conduca alla formazione dei parametri di forme non riducibili a coefficienti costanti, poichè in questo caso le equazioni (11') del $\S 3$ assumono una forma diversa. Il procedimento si deve dunque, per ora, ritenere applicabile soltanto a forme che possono essere ridotte, con opportune trasformazioni di variabili, ad avere tutti i coefficienti costanti. 